\title{
Journal of the American Chemical Society
}

Supporting Information to Accompany:

Partha Ghosh, Stephen D. Lotesta, Lawrence J. Williams ${ }^{*}$

Contribution from the Department of Chemistry and Chemical Biology, Rutgers, The State University of New Jersey, Piscataway, New Jersey 08854, USA

General: Starting materials, reagents and solvents were purchased from commercial suppliers (Aldrich, Acros, Lancaster, and Fischer) and used without further purification. Anhydrous THF, $\mathrm{Et}_{2} \mathrm{O}, \mathrm{CHCl}_{3}$, and DCM were obtained from a solvent purification system consisting of alumina based columns. All reactions were conducted in oven-dried $\left(135^{\circ} \mathrm{C}\right)$ glassware under an inert atmosphere of dry nitrogen. The progress of reactions were monitored by silica gel thin layer chromatography (tlc) plates (mesh size $60 \AA$ with fluorescent indicator, Sigma-Aldrich), visualized under UV and charred using cerium or anisaldehyde stain. Products were purified by flash column chromatography (FCC) on 120-400 mesh silica gel (Fisher). Infrared (FTIR) spectra were recorded on an ATI Mattson Genesis Series FT-Infrared spectrophotometer. Proton nuclear magnetic resonance spectra $\left({ }^{1} \mathrm{H}\right.$ NMR) were recorded on either a Varian-300 instrument (300 MHz), Varian-400 instrument (400 MHz), or a Varian-500 instrument (500 MHz). Chemical shifts are reported in ppm relative to tetramethylsilane (TMS) as the internal standard. Data is reported as follows: chemical shift, integration, multiplicity (s=singlet, d=doublet, $\mathrm{t}=$ triplet, $\mathrm{q}=$ quartet, br=broad, $\mathrm{m}=$ multiplet), and coupling constants $(\mathrm{Hz})$. Carbon nuclear magnetic resonance spectra $\left({ }^{13} \mathrm{C} \mathrm{NMR}\right)$ were recorded on either a Varian-300 instrument (75 MHz), Varian-400 instrument (100 MHz) or a Varian-500 instrument (125 MHz). Chemical shifts are reported in ppm relative to tetramethylsilane (TMS) as the internal standard. Optical rotations were recorded at $25^{\circ} \mathrm{C}$ using the sodium D line $(589 \mathrm{~nm})$, on a Perkin-Elmer 241 polarimeter. Mass spectra were recorded on a Finnigan LCQ-DUO mass spectrometer. 


\section{$\underline{\text { Rationale for Stereochemical Outcome of Allene Oxidation }}$}<smiles></smiles>

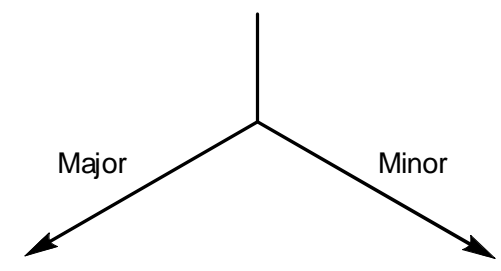<smiles>[3H]C=C1O[C@@]1([CH])[S-]</smiles><smiles>[Si]C1([Si])O/C1=C/I</smiles>
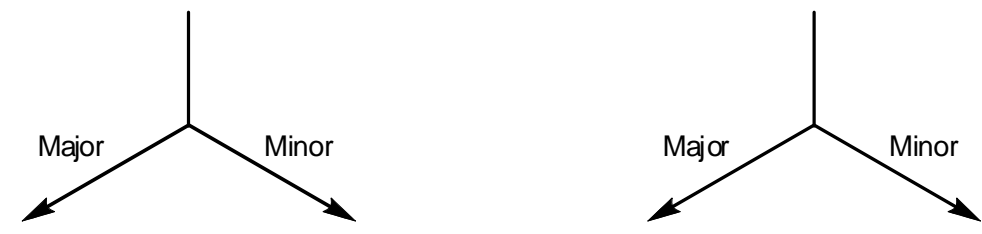<smiles>[CH][C@H]1O[C@@H]1[C@@H]1O[C@]1([CH])[SiH3]</smiles><smiles>[CH]C1([SiH3])OC12OC2([CH])[SiH3]</smiles><smiles></smiles>

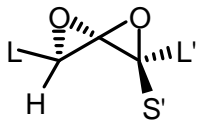

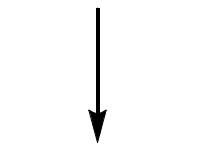
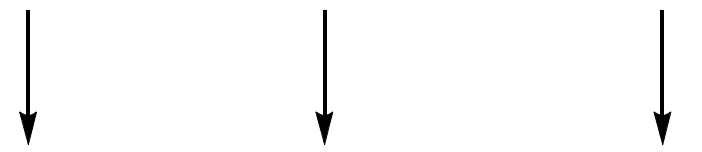<smiles>N[C@@H](I)C(=O)[C@](O)(S)I</smiles><smiles>N[C@@H](I)C(=O)[C@](O)(S)I</smiles>

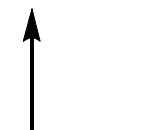

$\uparrow$

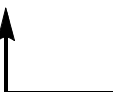

Enantiomers<smiles>N[C@@H](I)C(=O)C(O)(O)I</smiles>

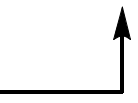

(n)

Enantiomers

$$
\mathrm{S}=\text { small, } \mathbf{L}=\text { large }
$$

\section{General Method for Determination of Diastereomeric Ratios}

Diastereomeric ratios for all spirodiepoxides (SDEs) were determined by ${ }^{1} \mathrm{H}$ NMR analysis $^{1}$ by comparison of the signals between 3.5-3.8 $\mathrm{ppm}$ and/or 1.19-1.21 ppm. The

\footnotetext{
${ }^{1}$ For examples of dr determinations of spirodiepoxides see: Crandall, J. K.; Batal, D. J.; Sebesta, D. P.; Ling, F. J. Org. Chem, 1991, 56, 1153
} 
selectivity of the $1^{\text {st }}$ oxidation (Table $2, \mathrm{r}_{1}$ ) was determined by analysis of the spirodiepoxide derived from allene $\mathbf{6 b}$ (see ${ }^{1} \mathrm{H}$ NMR of $\mathbf{6} \mathbf{b}^{\prime}$ on S-45). In all cases, the ratios of the SDEs matched the ratios of the cuprate addition products.

Diastereomeric ratios for cuprate addition products were determined by ${ }^{1} \mathrm{H}$ NMR analysis of the crude mixture by comparison of baseline resolved signals between 1.2-3.8 ppm. Due to inseparability of the diastereomers, only the minor isomer of the methyl addition product derived from allene 6a (Table 2, entry 1) was characterized (see S-10, S-52, S-53).

\section{Assignment of Absolute Stereochemistry}

a) The methyl addition product derived from allene 6a (Table 2, entry 1) is a known compound whose spectral data matched identically to the compound from ref. 7 (see S-10).

b) A derivative of the methyl addition product derived from allene $\mathbf{6 c}$ (Table 2, entry 3) was synthesized and an X-ray crystal structure was obtained (see S-29).

c) Product 15, derived from allene 6d (Table 2, entry 4) was converted to known compound 17 whose spectral data matched identically to the compound from ref. 11 (see S-28)

\section{General procedure for preparation of SDE from allenes}

To a solution of freshly prepared dimethyldioxirane (DMDO) in $\mathrm{CHCl}_{3}{ }^{2}(\sim 0.20 \mathrm{M}, 3$ equiv.) was added the allene in $\mathrm{CHCl}_{3}$ dropwise at $-40^{\circ} \mathrm{C}$. The reaction was stirred under nitrogen and

${ }^{2}$ Gibert, M.; Ferrer, M.; Sanchez-Baeza, F.; Messeguer, A. Tetrahedron. 1997, 53, 8643. 
allowed to warm to $\mathrm{rt}$ over $2 \mathrm{~h}$. The solvent was evaporated and the resulting SDE was dried under vacuum and used for the next step without further purification.

\section{Table (1) General Procedure (entry 1-5, 8):}

Allenes $(50 \mathrm{mg})$ were oxidized to SDE according to the general procedure above. Cuprates were prepared according to the literature procedures. ${ }^{3}$ SDEs were added to the cuprates (2.5 equiv.) at $-78^{\circ} \mathrm{C}$ and allowed to warm to $0^{\circ} \mathrm{C}$ over $2 \mathrm{~h}$. (for entries 4 and 8 the reactions were warmed to $\mathrm{rt}$ and stirred for an additional $1 \mathrm{~h}$ ). Upon completion by tlc, the reactions were quenched with saturated aq. $\mathrm{NH}_{4} \mathrm{Cl}$ and extracted with $\mathrm{Et}_{2} \mathrm{O}$. The $\mathrm{Et}_{2} \mathrm{O}$ layer was washed with water, brine and dried over anhydrous $\mathrm{MgSO}_{4}$. Evaporation of solvent and $\mathrm{FCC}$ gave compounds $\mathbf{7}$ and $\mathbf{8}$ as an inseparable mixture. Their individual yields are based on their ratios by ${ }^{1} \mathrm{H}$ NMR.

\section{General procedures for cuprate additions}

\section{Procedure A:}

A suspension of $\mathrm{CuCN}$ (activated by a gentle flame under high vacuum) in anhydrous $\mathrm{Et}_{2} \mathrm{O}$ was degassed for 2 min with argon. The suspension was cooled to $-10^{\circ} \mathrm{C}$ and a solution of $1.6 \mathrm{M}$ $\mathrm{MeLi}$ in $\mathrm{Et}_{2} \mathrm{O}$ was added dropwise. The reaction mixture was warmed to $0^{\circ} \mathrm{C}$ at which point it became a colorless homogeneous solution. The solution was cooled back to $-10^{\circ} \mathrm{C}$ and the SDE in anhydrous $\mathrm{Et}_{2} \mathrm{O}$ was added dropwise. The reaction was allowed to warm to rt over 30 min and monitored by tlc. Upon completion by tlc, the reaction was quenched with saturated aq. $\mathrm{NH}_{4} \mathrm{Cl}$

\footnotetext{
${ }^{3}$ For entries 1 and 3 see: Baldwin, I.C.; Beckett, R.P.; Williams, J.M.J. Synthesis 1996, 34. For entry 2 see: Hrubiec, R.T.; J. Org. Chem. 1984, 49, 385. For entry 4 see: Elsevier, C.J..; Vermeer, P. J. Org. Chem. 1989, 54, 3726. For entry 5 see: Lipshutz B.H.; Wilhelm R.S.; Kozlowski J.A..; Parker D. J. Org. Chem. 1984, 49, 3928.

For entry 8 see: Meyers A.I..; Snyder L. J. Org. Chem. 1992, 57, 3814.
} 
( $2 \mathrm{ml})$, extracted with $\mathrm{Et}_{2} \mathrm{O}(3 \times 5 \mathrm{ml})$, dried over anhydrous $\mathrm{MgSO}_{4}$ and evaporated to give crude product, which upon further purification by FCC gave the $\alpha$-hydroxy ketone.

\section{Procedure B:}

A suspension of $\mathrm{CuCN}$ (activated by a gentle flame under high vacuum) in anhydrous $\mathrm{Et}_{2} \mathrm{O}$ was degassed for 2 min with argon. The suspension was cooled to $-78^{\circ} \mathrm{C}$ and a solution of $1.6 \mathrm{M} \mathrm{n}$ $\mathrm{BuLi}$ in hexane was added dropwise. The reaction mixture was warmed to $-20^{\circ} \mathrm{C}$ at which point it became a homogeneous solution. The solution was cooled back to $-78^{\circ} \mathrm{C}$ and the SDE in anhydrous $\mathrm{Et}_{2} \mathrm{O}$ was added dropwise. The reaction was allowed to warm to $\mathrm{rt}$ over $2 \mathrm{~h}$ and monitored by tlc. Upon completion by tlc, the reaction was quenched with saturated aq. $\mathrm{NH}_{4} \mathrm{Cl}$ $(2 \mathrm{ml})$, extracted with $\mathrm{Et}_{2} \mathrm{O}(3 \times 5 \mathrm{ml})$, dried over anhydrous $\mathrm{MgSO}_{4}$ and evaporated to give crude product, which upon further purification by FCC gave the $\alpha$-hydroxy ketone.

\section{Procedure C:}

A suspension of $\mathrm{CuCN}$ (activated by a gentle flame under high vacuum) in anhydrous $\mathrm{Et}_{2} \mathrm{O}$ was degassed for 2 min with argon. The suspension was cooled to $-78^{\circ} \mathrm{C}$ and a solution of $1.0 \mathrm{M}$ $\mathrm{TMSCH}_{2} \mathrm{Li}$ in pentane was added dropwise. The reaction mixture was warmed to $\mathrm{rt}$ at which point it became a homogeneous solution. The solution was cooled back to $-78^{\circ} \mathrm{C}$ and the SDE in anhydrous $\mathrm{Et}_{2} \mathrm{O}$ was added dropwise. The reaction was allowed to warm to $\mathrm{rt}$ over $2 \mathrm{~h}$ and monitored by tlc. Upon completion by tlc, the reaction was quenched with saturated aq. $\mathrm{NH}_{4} \mathrm{Cl}$ $(2 \mathrm{ml})$, extracted with $\mathrm{Et}_{2} \mathrm{O}(3 \times 5 \mathrm{ml})$, dried over anhydrous $\mathrm{MgSO}_{4}$ and evaporated to give crude product, which upon further purification by FCC gave the $\alpha$-hydroxy ketone.

\section{Procedure D:}


A suspension of $\mathrm{CuCN}$ (activated by a gentle flame under high vacuum) in anhydrous $\mathrm{Et}_{2} \mathrm{O}$ was degassed for 2 min with argon. The suspension was cooled to $-78^{\circ} \mathrm{C}$ and a solution of $2.0 \mathrm{M} \mathrm{PhLi}$ in dibutylether was added dropwise. The reaction mixture was warmed to $0^{\circ} \mathrm{C}$ at which point it became a homogeneous solution. The solution was cooled back to $-78^{\circ} \mathrm{C}$ and the SDE in anhydrous $\mathrm{Et}_{2} \mathrm{O}$ was added dropwise. The reaction was allowed to warm to $\mathrm{rt}$ over $2 \mathrm{~h}$ and monitored by tlc. Upon completion by tlc, the reaction was quenched with saturated aq. $\mathrm{NH}_{4} \mathrm{Cl}$ (2 ml), extracted with $\mathrm{Et}_{2} \mathrm{O}(3 \times 5 \mathrm{ml})$, dried over anhydrous $\mathrm{MgSO}_{4}$ and evaporated to give crude product, which upon further purification by FCC gave the $\alpha$-hydroxy ketone.

\section{Procedure E:}

A suspension of $\mathrm{CuI}$ in anhydrous $\mathrm{Et}_{2} \mathrm{O}$ was degassed with argon for 2 min and cooled to $-78^{\circ} \mathrm{C}$. A solution of $2.0 \mathrm{M} \mathrm{PhLi}$ in dibutylether was added dropwise to the suspension at $-78^{\circ} \mathrm{C}$. The reaction mixture was warmed to $0^{\circ} \mathrm{C}$ at which point a dark black solution was formed. The solution was cooled back to $-78^{\circ} \mathrm{C}$ and the $\mathrm{SDE}$ in anhydrous $\mathrm{Et}_{2} \mathrm{O}$ was added dropwise. The reaction mixture was allowed to warm to rt over $2 \mathrm{~h}$ and monitored by tlc. Upon completion by tlc, the reaction was quenched with saturated aq. $\mathrm{NH}_{4} \mathrm{Cl}(2 \mathrm{ml})$, extracted with $\mathrm{Et}_{2} \mathrm{O}(3 \times 5 \mathrm{ml})$, dried over anhydrous $\mathrm{MgSO}_{4}$ and evaporated to give crude product, which upon further purification by FCC gave the $\alpha$-hydroxy ketone.

\section{Procedure F:}

A suspension of $\mathrm{CuCN}$ (activated by a gentle flame under high vacuum) in anhydrous $\mathrm{Et}_{2} \mathrm{O}$ was degassed for 2 min with argon. The suspension was cooled to $-10^{\circ} \mathrm{C}$ and a solution of $1.0 \mathrm{M}$ $\mathrm{MeMgBr}$ in dibutylether was added dropwise. The reaction mixture was warmed to $0^{\circ} \mathrm{C}$ at which point it became a pale yellow homogeneous solution. The solution was cooled back to $-10^{\circ} \mathrm{C}$ and 
the $\mathrm{SDE}$ in anhydrous $\mathrm{Et}_{2} \mathrm{O}$ was added dropwise. The reaction was allowed to warm to rt over 30 min and monitored by tlc. Upon completion by tlc, the reaction was quenched with saturated aq. $\mathrm{NH}_{4} \mathrm{Cl}(2 \mathrm{ml})$, extracted with $\mathrm{Et}_{2} \mathrm{O}(3 \times 5 \mathrm{ml})$, dried over anhydrous $\mathrm{MgSO}_{4}$ and evaporated to give crude product, which upon further purification by FCC gave the $\alpha$-hydroxy ketone.

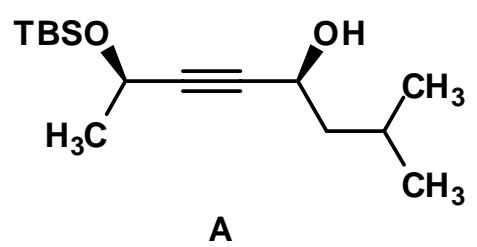

A suspension of $\mathrm{Zn}(\mathrm{OTf})_{2}(1.74 \mathrm{~g}, 4.8 \mathrm{mmol}),(-)-\mathrm{N}-$ Methylephedrine (860 mg, $\left.4.8 \mathrm{mmol}\right)$ and $\mathrm{Et}_{3} \mathrm{~N}$ $(6.69 \mathrm{ml}, 4.80 \mathrm{mmol})$ in $20 \mathrm{ml}$ of toluene was stirred at room temperature for $30 \mathrm{~min}$. TBS-protected (R)-3-butyn-2-ol ${ }^{4}(800 \mathrm{mg}, 4.34 \mathrm{mmol}$ ) was added in one portion and the reaction stirred for $30 \mathrm{~min}$. Isovaleraldehyde (380 mg, $4.41 \mathrm{mmol}$ ) was added in one portion and the reaction was stirred for an additional $12 \mathrm{~h}$ at $\mathrm{rt}$ and monitored by tlc. Upon completion by tlc, the reaction was quenched with saturated aq. $\mathrm{NH}_{4} \mathrm{Cl}(10 \mathrm{ml})$ and extracted with $\mathrm{Et}_{2} \mathrm{O}(3 \times 15 \mathrm{ml})$. The organic layer was dried over anhydrous MgSO4 and evaporated to give the crude product. FCC using 10\% ethyl acetate-hexane gave $856 \mathrm{mg}$ of propargylic alcohol $\mathbf{A}(73 \%,>95: 5 \mathrm{dr})$ as a clear colorless oil $\left(\mathrm{R}_{\mathrm{f}}=0.50\right.$ in $15 \%$ ethyl acetate-hexanes). $[\alpha]_{\mathrm{D}}+33.0(c=0.01, \mathrm{MeOH}) . \mathrm{IR} v_{\max }$ (neat) $/ \mathrm{cm}^{-1} 3350,1463,1255,1102$, $836, \delta_{\mathrm{H}}\left(400 \mathrm{MHz}, \mathrm{CDCl}_{3}\right) 4.55(1 \mathrm{H}, \mathrm{qd}, \mathrm{J}=6.4,1.2 \mathrm{~Hz}), 4.43(1 \mathrm{H}, \mathrm{q}, \mathrm{J}=6.4 \mathrm{~Hz}), 1.85-1.80(1 \mathrm{H}, \mathrm{m})$, $1.71(1 \mathrm{H}, \mathrm{d}, \mathrm{J}=5.2 \mathrm{~Hz}), 1.66-1.50(2 \mathrm{H}, \mathrm{m}), 1.40(3 \mathrm{H}, \mathrm{d}, \mathrm{J}=6.4 \mathrm{~Hz}), 0.94(3 \mathrm{H}, \mathrm{d}, \mathrm{J}=6.8 \mathrm{~Hz}), 0.92(3 \mathrm{H}$, d, J=7.2 Hz), $0.90(9 \mathrm{H}, \mathrm{s}), 0.12(3 \mathrm{H}, \mathrm{s}), 0.11(3 \mathrm{H}, \mathrm{s}) ; \delta_{\mathrm{c}}\left(100 \mathrm{MHz}, \mathrm{CDCl}_{3}\right)$ 87.4, 84.3, 61.0, 58.9, 46.7, 25.8, 25.4, 24.7, $22.5(2), 18.2,-4.6,-4.9 ; \mathrm{m} / \mathrm{z}$ (ESIMS) $293.3(\mathrm{M}+23)^{+}$.

\footnotetext{
${ }^{4}$ Obtained in $>95 \%$ yield by silylation of commercial (R)-3-butyn-2-ol (Aldrich, 99\% ee) with TBS-Cl / Imidazole
} 


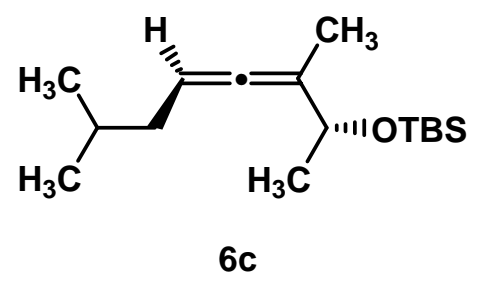

To a solution of propargylic alcohol $\mathbf{A}(315 \mathrm{mg}, 1.16 \mathrm{mmol})$ in dry $\mathrm{CH}_{2} \mathrm{Cl}_{2}(5 \mathrm{ml})$ was added $\mathrm{Et}_{3} \mathrm{~N}(0.2 \mathrm{ml}, 1.43 \mathrm{mmol})$ dropwise. The solution was cooled to $0^{\circ} \mathrm{C}$ and $\mathrm{MsCl}(0.11 \mathrm{ml}$, $1.42 \mathrm{mmol}$ ) was added dropwise and the reaction stirred for $1 \mathrm{~h}$ at $0^{\circ} \mathrm{C}$. The reaction was quenched by the addition of water $(1 \mathrm{ml})$. The organic layer was separated, washed with water $(2 \times 5 \mathrm{ml})$ and dried over anhydrous $\mathrm{MgSO}_{4}$. The solvent was evaporated and the crude mesylate was dried under vacuum and used for the next step without further purification.

To a suspension of $\mathrm{CuCN}(160 \mathrm{mg}, 1.79 \mathrm{mmol})$ in anhydrous $\mathrm{Et}_{2} \mathrm{O}(10 \mathrm{ml})$ at $-40^{\circ} \mathrm{C}$ was added 1.6 M MeLi in $\mathrm{Et}_{2} \mathrm{O}(1.10 \mathrm{ml}, 1.76 \mathrm{mmol})$. The solution was warmed to $0^{0} \mathrm{C}$ and stirred for $10 \mathrm{~min}$ at which point a colorless homogeneous solution had formed. The reaction mixture was then cooled back to $-40^{\circ} \mathrm{C}$ and the above mesylate in $5 \mathrm{ml}$ of anhydrous $\mathrm{Et}_{2} \mathrm{O}$ was added dropwise. The reaction mixture was allowed to warm to rt over $1 \mathrm{~h}$ and monitored by tlc. Upon completion by tlc, the reaction was quenched with saturated aq. $\mathrm{NH}_{4} \mathrm{Cl}(5 \mathrm{ml})$ and extracted with $\mathrm{Et}_{2} \mathrm{O}(3 \times 10 \mathrm{ml})$. The $\mathrm{Et}_{2} \mathrm{O}$ layer was washed with saturated aq. $\mathrm{NH}_{4} \mathrm{Cl}(10 \mathrm{ml})$, water $(10 \mathrm{ml})$, brine $(10 \mathrm{ml})$ and dried over anhydrous $\mathrm{MgSO}_{4}$. Evaporation of solvent and $\mathrm{FCC}$ purification using hexane gave $290 \mathrm{mg}$ of allene $\mathbf{6 c}(93 \%)$ as a clear colorless oil $\left(\mathrm{R}_{\mathrm{f}}=0.90\right.$ in hexanes). $[\alpha]_{\mathrm{D}}$ $+36.0(c=0.01, \mathrm{MeOH}) . \mathrm{IR} v_{\max }$ (neat) $/ \mathrm{cm}^{-1} 1966,1255,1463,1084,834 ; \delta_{\mathrm{H}}(400 \mathrm{MHz}$, $\left.\mathrm{CDCl}_{3}\right)$ 5.06-4.94 (1H, m), $4.28(1 \mathrm{H}, \mathrm{q}, \mathrm{J}=6.4 \mathrm{~Hz}), 1.87\left(2 \mathrm{H}, \mathrm{dd}, \mathrm{J}_{1}=7.2 \mathrm{~Hz}, \mathrm{~J}_{2}=7.2 \mathrm{~Hz}\right), 1.66$ $(3 \mathrm{H}, \mathrm{d}, \mathrm{J}=2.8 \mathrm{~Hz}), 1.62-1.68(1 \mathrm{H}, \mathrm{m}), 1.23(3 \mathrm{H}, \mathrm{d}, \mathrm{J}=6.4 \mathrm{~Hz}), 0.91(6 \mathrm{H}, \mathrm{d}, \mathrm{J}=6.4 \mathrm{~Hz}), 0.89(9 \mathrm{H}$, s), $0.06(6 \mathrm{H}, \mathrm{s}) ; \delta_{\mathrm{c}}\left(100 \mathrm{MHz}, \mathrm{CDCl}_{3}\right) 200.8,102.6,89.6,70.3,38.5,28.6,25.9(3), 22.9,22.3$, 22.2, 18.2, 13.9, -4.7, -4.9; $\mathrm{m} / z$ (ESIMS) $269.1(\mathrm{M}+1)^{+}$. 


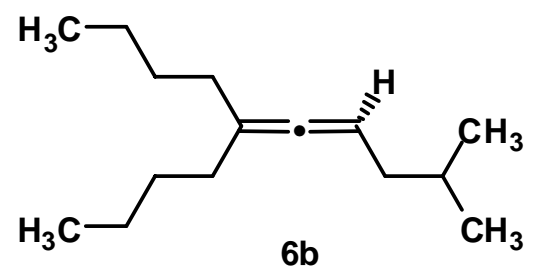

To a solution of known 2-methyldec-5-yn-4-ol ${ }^{5}$ (1.30 gm, $\left.7.73 \mathrm{mmol}\right)$ in $20 \mathrm{ml}$ dry $\mathrm{CH}_{2} \mathrm{Cl}_{2}$ was added $\mathrm{Et}_{3} \mathrm{~N}(1.30 \mathrm{ml}, 9.3 \mathrm{mmol})$ dropwise. The solution was cooled to $0^{\circ} \mathrm{C}$ and $\mathrm{MsCl}(0.70 \mathrm{ml}, 9.0 \mathrm{mmol})$ was added dropwise and the reaction stirred for $1 \mathrm{~h} \mathrm{at} 0^{\circ} \mathrm{C}$. The reaction was quenched by the addition of water $(1 \mathrm{ml})$. The organic layer was separated, washed with water $(2 \times 5 \mathrm{ml})$ and dried over anhydrous $\mathrm{MgSO}_{4}$. The solvent was evaporated and the crude mesylate was dried under vacuum and used for the next step without further purification.

To a suspension of $\mathrm{CuCN}(860 \mathrm{mg}, 9.6 \mathrm{mmol})$ in anhydrous $\mathrm{Et}_{2} \mathrm{O}(50 \mathrm{ml})$ at $-40^{\circ} \mathrm{C}$ was added $1.6 \mathrm{M} \mathrm{n}$-BuLi in hexane $(6.0 \mathrm{ml}, 9.6 \mathrm{mmol})$. The solution was warmed to $-20^{\circ} \mathrm{C}$ and stirred for 5 min at which point a homogeneous solution had formed. The reaction mixture was then cooled back to $-40^{\circ} \mathrm{C}$ and the above mesylate in anhydrous $\mathrm{Et}_{2} \mathrm{O}(5 \mathrm{ml})$ was added dropwise. The reaction mixture was allowed to warm to rt over $1 \mathrm{~h}$ and monitored by tlc. Upon completion by tlc, the reaction was quenched with saturated aq. $\mathrm{NH}_{4} \mathrm{Cl}(10 \mathrm{ml})$ and extracted with $\mathrm{Et}_{2} \mathrm{O}(3 \times 15 \mathrm{ml})$. The $\mathrm{Et}_{2} \mathrm{O}$ layer was washed with saturated aq. $\mathrm{NH}_{4} \mathrm{Cl}(20 \mathrm{ml})$, water $(20$ $\mathrm{ml})$, brine $(20 \mathrm{ml})$ and dried over anhydrous $\mathrm{MgSO}_{4}$. Evaporation of solvent and $\mathrm{FCC}$ purification using hexane gave $1.47 \mathrm{~g}$ of allene $\mathbf{6 b}(92 \%)$ as a clear colorless oil $\left(\mathbf{R}_{\mathrm{f}}=0.90\right.$ in hexanes). IR $v_{\max }$ (neat) $/ \mathrm{cm}^{-1} 1961,1466,1379 ; \delta_{\mathrm{H}}\left(400 \mathrm{MHz}, \mathrm{CDCl}_{3}\right) 5.06-4.96(1 \mathrm{H}, \mathrm{m}), 1.91$ $(4 \mathrm{H}, \mathrm{td}, \mathrm{J}=7.2,2.4 \mathrm{~Hz}), 1.87(1 \mathrm{H}, \mathrm{d}, \mathrm{J}=7.2 \mathrm{~Hz}), 1.85(1 \mathrm{H}, \mathrm{d}, \mathrm{J}=6.8 \mathrm{~Hz}), 1.68-1.58(1 \mathrm{H}, \mathrm{m})$, 1.44-1.28 (8H, m), $0.92(6 \mathrm{H}, \mathrm{d}, \mathrm{J}=6.4 \mathrm{~Hz}), 0.89(6 \mathrm{H}, \mathrm{t}, \mathrm{J}=7.2 \mathrm{~Hz}) ; \delta_{\mathrm{c}}\left(100 \mathrm{MHz}, \mathrm{CDCl}_{3}\right) 201.4$,

${ }^{5}$ Ma, S.; Wu, B.; Jiang, X.; Zhao, S. J. Org. Chem. 2005, 70, 2568-2575. 
103.3, 90.2, 39.3, 32.5(2), 30.0 (2), 28.7, 22.5 (2), 22.3(2), 14.0 (2); m/z (ESIMS) 231.2 $(\mathrm{M}+23)^{+}$.

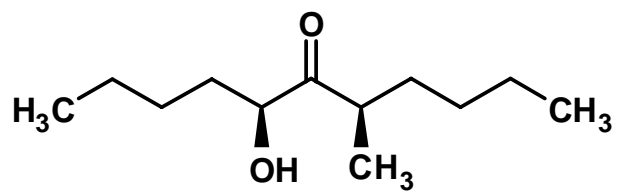

Known allene $\mathbf{6 a}^{6}$ (60 $\left.\mathrm{mg}, 0.670 \mathrm{mmol}\right)$ was converted to the SDE using the general procedure. Procedure A was employed using $\mathrm{CuCN}(60 \mathrm{mg}, 0.670 \mathrm{mmol}), 1.6 \mathrm{M} \mathrm{MeLi}$ in $\mathrm{Et}_{2} \mathrm{O}(0.41 \mathrm{ml}$, $0.656 \mathrm{mmol})$ and $10 \mathrm{ml} \mathrm{Et}{ }_{2} \mathrm{O}$. FCC using $2 \%$ ethyl acetate-hexane gave $39 \mathrm{mg}(74 \%, 2: 1 \mathrm{dr})$ of $\alpha$-hydroxy ketone as a clear colorless oil $\left(\mathrm{R}_{\mathrm{f}}=0.60\right.$ in $10 \%$ ethyl acetate-hexanes $)$. Major syn diastereomer spectra matched with that reported in literature. ${ }^{7}$ Spectral data for minor anti diastereomer: IR $v_{\max }$ (neat) $/ \mathrm{cm}^{-1} 3479,1708,1459,1052 ; \delta_{\mathrm{H}}\left(400 \mathrm{MHz}, \mathrm{CDCl}_{3}\right) 4.28-4.20(1 \mathrm{H}$, m), $3.51(1 \mathrm{H}, \mathrm{d}, \mathrm{J}=5.2 \mathrm{~Hz}), 2.78-2.68(1 \mathrm{H}, \mathrm{m}), 1.88-1.80(1 \mathrm{H}, \mathrm{m}), 1.66-1.56(1 \mathrm{H}, \mathrm{m}), 1.54-1.16$ $(10 \mathrm{H}, \mathrm{m}), 1.09(3 \mathrm{H}, \mathrm{d}, \mathrm{J}=6.4 \mathrm{~Hz}), 0.92(3 \mathrm{H}, \mathrm{t}, \mathrm{J}=7.2 \mathrm{~Hz}), 0.88(3 \mathrm{H}, \mathrm{t}, \mathrm{J}=7.2 \mathrm{~Hz}) ; \delta_{\mathrm{c}}(100 \mathrm{MHz}$, $\mathrm{CDCl}_{3}$ ) 216.5, 76.3, 41.2, 34.1, 33.2, 29.2, 27.3, 22.7, 22.5, 15.7, 13.9, 13.8; $\mathrm{m} / \mathrm{z}$ (ESIMS) 265.0 $(\mathrm{M}+23)^{+}$

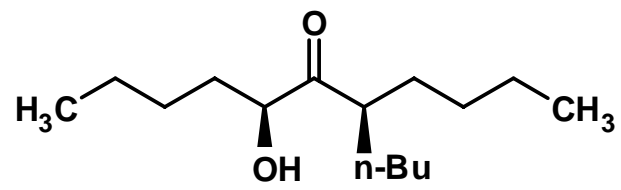

Known allene $\mathbf{6 a}^{6}$ (40 $\mathrm{mg}, 0.263 \mathrm{mmol}$ ) was converted to the SDE using the general procedure. Procedure B was employed using $\mathrm{CuCN}$ (60 mg, $0.670 \mathrm{mmol}), 1.6 \mathrm{M} \mathrm{n}-\mathrm{BuLi}$ in hexanes $(0.41$ $\mathrm{ml}, 0.656 \mathrm{mmol})$ and $10 \mathrm{ml} \mathrm{Et} 2 \mathrm{O}$. FCC using 2\% ethyl acetate-hexane gave $43 \mathrm{mg}(67 \%)$ of $\alpha$ hydroxy ketone as a clear colorless oil $\left(R_{\mathrm{f}}=0.65\right.$ in $10 \%$ ethyl acetate-hexanes). IR $v_{\max }$ (neat)

\footnotetext{
${ }^{6}$ Hailes, H.C.; Isaac, B.; Javaid, M.H. Syn. Comm. 2003, 33, 29.

${ }^{7}$ Gosmini, C.; Dubuffet, T.; Sauvêtre, R.; Normant, J.F. Tetrahedron: Asymmetry. 1991, 2, 223.
} 
$/ \mathrm{cm}^{-1} 3480,1706,1467,1050 ; \delta_{\mathrm{H}}\left(400 \mathrm{MHz}, \mathrm{CDCl}_{3}\right) 4.24-4.14(1 \mathrm{H}, \mathrm{m}), 3.48(1 \mathrm{H}, \mathrm{d}, \mathrm{J}=5.6)$, 2.70-2.60 (1H, m), 1.88-1.77 (1H, m), 1.73-1.63 (1H, m), 1.60-1.14 (16H, m), $0.92(3 \mathrm{H}, \mathrm{t}, \mathrm{J}=7.2$ $\mathrm{Hz}), 0.88(3 \mathrm{H}, \mathrm{t}, \mathrm{J}=7.2 \mathrm{~Hz}), 0.87(3 \mathrm{H}, \mathrm{t}, \mathrm{J}=7.2 \mathrm{~Hz}) ; \delta_{\mathrm{c}}\left(100 \mathrm{MHz}, \mathrm{CDCl}_{3}\right) 216.3,76.4,47.1$, 33.0, 32.8, 30.2, 29.8, 29.3, 27.5, 22.8, 22.4, $13.9(2), 13.8 ; \mathrm{m} / \mathrm{z}$ (ESIMS) $265.3(\mathrm{M}+23)^{+}$.

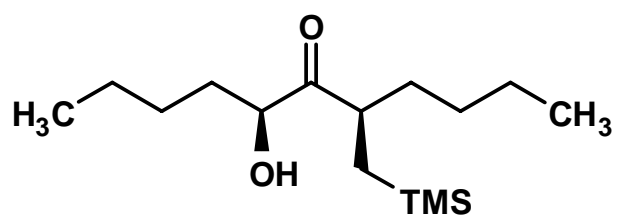

Known allene $\mathbf{6 a}^{6}(60 \mathrm{mg}, 0.394 \mathrm{mmol})$ was converted to the SDE using the general procedure. Procedure $\mathrm{C}$ was employed using $\mathrm{CuCN}(90 \mathrm{mg}, 1.00 \mathrm{mmol}), 1.0 \mathrm{M} \mathrm{TMSCH}_{2} \mathrm{Li}$ in pentane (1 $\mathrm{ml}, 1.00 \mathrm{mmol}$ ) and $15 \mathrm{ml} \mathrm{Et} 2 \mathrm{O}$. FCC using 2\% ethyl acetate-hexane gave $71 \mathrm{mg}(66 \% 2: 1 \mathrm{dr}$ ) of $\alpha$-hydroxy ketone as a clear colorless oil $\left(\mathrm{R}_{\mathrm{f}}=0.70\right.$ in $10 \%$ ethyl acetate-hexanes $)$. Spectral data for major isomer: IR $v_{\max }$ (neat) $/ \mathrm{cm}^{-1} 3480,1708,1249,859 ; \delta_{\mathrm{H}}\left(400 \mathrm{MHz}, \mathrm{CDCl}_{3}\right) 4.26-$ $4.17(0.66 \mathrm{H}, \mathrm{m}), 4.17-4.12(0.33 \mathrm{H}, \mathrm{m}), 3.47(0.33 \mathrm{H}, \mathrm{d}, \mathrm{J}=5.2 \mathrm{~Hz}), 3.41(0.66 \mathrm{H}, \mathrm{d}, \mathrm{J}=5.6 \mathrm{~Hz})$, 2.76-2.68 $(0.33 \mathrm{H}, \mathrm{m}), 2.68-2.59(0.66 \mathrm{H}, \mathrm{m}), 1.82-1.04(12 \mathrm{H}, \mathrm{m}), 0.87(3 \mathrm{H}, \mathrm{t}, \mathrm{J}=6.8 \mathrm{~Hz}), 0.84$ $(2 \mathrm{H}, \mathrm{t}, \mathrm{J}=6.8 \mathrm{~Hz}), 0.84(1 \mathrm{H}, \mathrm{t}, \mathrm{J}=6.8 \mathrm{~Hz}), 0.79-0.58(2 \mathrm{H}, \mathrm{m}),-0.01(6 \mathrm{H}, \mathrm{s}),-0.02(3 \mathrm{H}, \mathrm{s}) ; \delta_{\mathrm{c}}(100$ $\left.\mathrm{MHz}, \mathrm{CDCl}_{3}\right)$ 216.2, 215.9, 76.3, 74.9, 42.9, 42.7, 34.7, 33.6,33.2, 32.4, 29.9, 29.1, 27.7, 27.4, 22.7, 22.4, 22.4, 20.7, 17.8, 13.9, 13.8, -0.9, -1.0; $\mathrm{m} / \mathrm{z}$ (ESIMS) $295.2(\mathrm{M}+23)^{+}$.

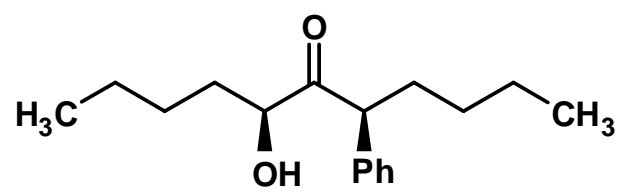

Known allene $\mathbf{6 a}^{6}$ (60 $\mathrm{mg}, 0.394 \mathrm{mmol}$ ) was converted to the SDE using the general procedure. Procedure D was employed using CuCN (108 mg, $1.20 \mathrm{mmol}), 2.0 \mathrm{M}$ in dibutylether PhLi (0.6 
$\mathrm{ml}, 1.2 \mathrm{mmol})$ and $10 \mathrm{ml} \mathrm{Et}{ }_{2} \mathrm{O}$. FCC using 2\% ethyl acetate-hexane gave $49.5 \mathrm{mg}(59 \%, 2: 1 \mathrm{dr})$ of $\alpha$-hydroxy ketone as a clear colorless oil $\left(R_{\mathrm{f}}=0.70\right.$ in $10 \%$ ethyl acetate-hexanes $)$. Spectral data for major isomer: IR $v_{\max }$ (neat) $/ \mathrm{cm}^{-1} 3482,1708,1455,700 ; \delta_{\mathrm{H}}\left(400 \mathrm{MHz}, \mathrm{CDCl}_{3}\right) 7.34-$ $7.23(3 \mathrm{H}, \mathrm{m}), 7.20-7.15(2 \mathrm{H}, \mathrm{m}), 4.15-4.07(1 \mathrm{H}, \mathrm{m}), 3.80(1 \mathrm{H}, \mathrm{t}, \mathrm{J}=7.2 \mathrm{~Hz}), 3.29(1 \mathrm{H}, \mathrm{d}, \mathrm{J}=5.2$ Hz), 2.12-2.05 (1H, m), 1.88-1.68 (2H, m), 1.58-1.05 (9H, m), $0.91(3 \mathrm{H}, \mathrm{t}, \mathrm{J}=7.2 \mathrm{~Hz}), 0.86(3 \mathrm{H}$, $\mathrm{t}, \mathrm{J}=7.2 \mathrm{~Hz}) ; \delta_{\mathrm{c}}\left(100 \mathrm{MHz}, \mathrm{CDCl}_{3}\right) 212.2,138.3,129.0,128.2,128.1,127.5,74.7,54.1,33.4$, 31.8, 29.6, 27.0, 22.5, 22.4, 13.9, 13.8; $\mathrm{m} / \mathrm{z}(\mathrm{ESIMS}) 285.2(\mathrm{M}+23)^{+}$.

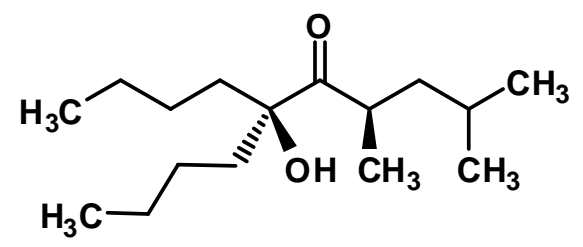

Allene 6b (40 mg, $0.192 \mathrm{mmol}$ ) was converted to the SDE using the general procedure. Procedure A was employed using $\mathrm{CuCN}$ (41 mg, $0.480 \mathrm{mmol}), 1.6 \mathrm{M} \mathrm{MeLi}$ in $\mathrm{Et}_{2} \mathrm{O}(0.3 \mathrm{ml}$, $0.480 \mathrm{mmol}$ ) and $10 \mathrm{ml} \mathrm{Et}_{2} \mathrm{O}$. FCC using 2\% ethyl acetate-hexane gave $42 \mathrm{mg}(85 \%)$ of an inseparable mixture of methyl addition and reduced product in a 20:1 ratio as a clear colorless oil $\left(\mathrm{R}_{\mathrm{f}}=0.65\right.$ in $10 \%$ ethyl acetate-hexanes $)$. Spectral data for methyl addition product: IR $v_{\max }$ (neat) $/ \mathrm{cm}^{-1} 3479,1699,1467,1054 ; \delta_{\mathrm{H}}\left(400 \mathrm{MHz}, \mathrm{CDCl}_{3}\right) 3.75(1 \mathrm{H}, \mathrm{s}), 3.03-2.94(1 \mathrm{H}, \mathrm{m})$, 1.77-1.62 (4H, m), 1.55-1.20 (8H, m), $1.06(3 \mathrm{H}, \mathrm{d}, \mathrm{J}=6.4 \mathrm{~Hz}), 1.03-0.94(2 \mathrm{H}, \mathrm{m}), 0.92(3 \mathrm{H}, \mathrm{d}$, $\mathrm{J}=6.4 \mathrm{~Hz}), 0.88(3 \mathrm{H}, \mathrm{d}, \mathrm{J}=6.0 \mathrm{~Hz}), 0.88(6 \mathrm{H}, \mathrm{t}, \mathrm{J}=6.8 \mathrm{~Hz}) ; \delta_{\mathrm{c}}\left(100 \mathrm{MHz}, \mathrm{CDCl}_{3}\right) 218.9,82.2$, 42.5, 37.8 (2), 36.8, 25.7, 25.2, 23.7, 23.0, 21.5, 16.9, 13.9 (2); $m / z$ (ESIMS) $279.3(\mathrm{M}+23)^{+}$. 


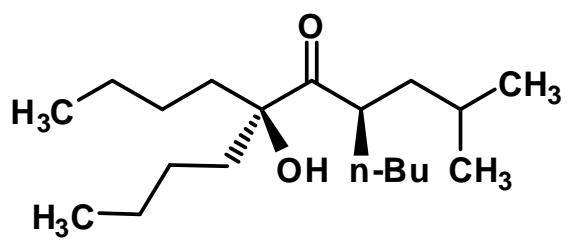

Allene 6b (40 mg, $0.192 \mathrm{mmol}$ ) was converted to the SDE using the general procedure. Procedure B was employed using $\mathrm{CuCN}(41 \mathrm{mg}, 0.480 \mathrm{mmol}), 1.6 \mathrm{M} \mathrm{n}-\mathrm{BuLi}$ in hexane $(0.3 \mathrm{ml}$, $0.480 \mathrm{mmol})$ and $10 \mathrm{ml} \mathrm{Et}{ }_{2} \mathrm{O}$. FCC using $2 \%$ ethyl acetate-hexane gave $41 \mathrm{mg}(72 \%)$ of $\alpha$ hydroxy ketone as a clear colorless oil $\left(R_{f}=0.70\right.$ in $10 \%$ ethyl acetate-hexanes). IR $v_{\max }$ (neat) $/ \mathrm{cm}^{-1} 3477,1694,1467 ; \delta_{\mathrm{H}}\left(400 \mathrm{MHz}, \mathrm{CDCl}_{3}\right) 3.52(1 \mathrm{H}, \mathrm{s}), 2.02-2.92(1 \mathrm{H}, \mathrm{m}), 1.78-1.52(6 \mathrm{H}$, m), $1.45-1.20(11 \mathrm{H}, \mathrm{m}), 1.12-1.00(2 \mathrm{H}, \mathrm{m}), 0.89(6 \mathrm{H}, \mathrm{d}, \mathrm{J}=6.8 \mathrm{~Hz}), 0.89(9 \mathrm{H}, \mathrm{t}, \mathrm{J}=7.6 \mathrm{~Hz}) ; \delta_{\mathrm{c}}$ $\left(100 \mathrm{MHz}, \mathrm{CDCl}_{3}\right)$ 218.0, 81.8, 42.4, 40.8, 37.4, 37.3, 31.3, 29.3, 25.7, 25.6, 25.5, 23.2, 23.0 (2), 22.9, 22.2, 14.0, $13.9(2) ; \mathrm{m} / z$ (ESIMS) $321.4(\mathrm{M}+23)^{+}$.

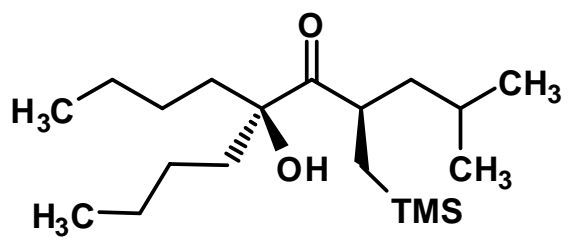

Allene 6b (40 mg, $0.192 \mathrm{mmol}$ ) was converted to the SDE using the general procedure. Procedure $\mathrm{C}$ was employed using $\mathrm{CuCN}(41 \mathrm{mg}, 0.480 \mathrm{mmol}), 1.0 \mathrm{M} \mathrm{TMSCH}_{2} \mathrm{Li}$ in pentane $(0.48 \mathrm{ml}, 0.480 \mathrm{mmol})$ and $10 \mathrm{ml} \mathrm{Et} 2 \mathrm{O}$. FCC using 2\% ethyl acetate-hexane gave $41 \mathrm{mg}(65 \%)$ of $\alpha$-hydroxy ketone as a clear colorless oil $\left(\mathrm{R}_{\mathrm{f}}=0.80\right.$ in $10 \%$ ethyl acetate-hexanes). IR $v_{\max }$ (neat) $/ \mathrm{cm}^{-1} 3485,1694,1467,1249 ; \delta_{\mathrm{H}}\left(400 \mathrm{MHz}, \mathrm{CDCl}_{3}\right) 3.339(1 \mathrm{H}, \mathrm{s}), 3.12-3.04(1 \mathrm{H}, \mathrm{m})$, 1.77-1.54 (5H, m), 1.44-1.22 (7H, m), 1.19-1.04 (3H, m), $0.89(6 \mathrm{H}, \mathrm{d}, \mathrm{J}=6.0 \mathrm{~Hz}), 0.89(6 \mathrm{H}, \mathrm{t}, \mathrm{J}=$ $7.2 \mathrm{~Hz}), 0.63-0.55(2 \mathrm{H}, \mathrm{m}), 0.06(9 \mathrm{H}, \mathrm{s}) ; \delta_{\mathrm{c}}\left(100 \mathrm{MHz}, \mathrm{CDCl}_{3}\right)$ 218.1, 81.7, 42.9, 38.5, 37.6, 37.3, 26.1, 25.7, 25.4, 23.4, 23.0, 22.3, 20.4, 14.0, $-0.4 ; \mathrm{m} / z$ (ESIMS) $351.3(\mathrm{M}+23)^{+}$. 


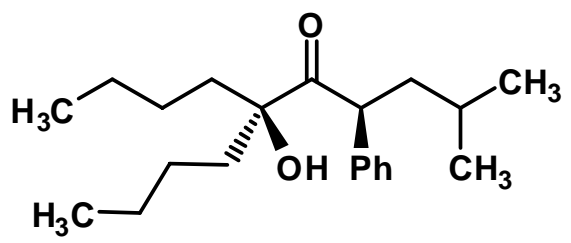

Allene 6b (40 mg, $0.192 \mathrm{mmol}$ ) was converted to the SDE using the general procedure. Procedure E was employed using CuI (110 mg, $0.578 \mathrm{mmol}), 2.0 \mathrm{M}$ PhLi in dibutylether $(0.58$ $\mathrm{ml}, 1.16 \mathrm{mmol})$ and $15 \mathrm{ml} \mathrm{Et}{ }_{2} \mathrm{O}$. FCC using $2 \%$ ethyl acetate-hexane gave $43 \mathrm{mg}(71 \%)$ of $\alpha$ hydroxy ketone as a clear colorless oil $\left(R_{\mathrm{f}}=0.70\right.$ in $10 \%$ ethyl acetate-hexanes). IR $v_{\max }$ (neat) $/ \mathrm{cm}^{-1} 3485,1699,1467 ; \delta_{\mathrm{H}}\left(400 \mathrm{MHz}, \mathrm{CDCl}_{3}\right) 7.32-7.28(4 \mathrm{H}, \mathrm{m}), 7.25-7.21(1 \mathrm{H}, \mathrm{m}), 4.116(1 \mathrm{H}$, dd $\left.\mathrm{J}_{1}=5.2,4.4 \mathrm{~Hz}\right), 3.62(1 \mathrm{H}, \mathrm{s}), 1.99-1.90(1 \mathrm{H}, \mathrm{m}), 1.84-1.66(2 \mathrm{H}, \mathrm{m}), 1.60-1.48(3 \mathrm{H}, \mathrm{m}), 1.44-$ $1.22(5 \mathrm{H}, \mathrm{m}), 1.16-1.04(1 \mathrm{H}, \mathrm{m}), 1.02-0.92(1 \mathrm{H}, \mathrm{m}), 0.892(3 \mathrm{H}, \mathrm{d}, \mathrm{J}=6.4 \mathrm{~Hz}), 0.890(3 \mathrm{H}, \mathrm{t}, \mathrm{J}=$ $7.2 \mathrm{~Hz}), 0.835(3 \mathrm{H}, \mathrm{J}=6.4 \mathrm{~Hz}), 0.78-0.68(1 \mathrm{H}, \mathrm{m}), 0.542(3 \mathrm{H}, \mathrm{t}, \mathrm{J}=7.2 \mathrm{~Hz}), 0.18-0.07(1 \mathrm{H}, \mathrm{m})$; $\delta_{\mathrm{c}}\left(100 \mathrm{MHz}, \mathrm{CDCl}_{3}\right) 214.8,138.0,128.6(2), 128.5$ (2), 127.2, 82.8, 49.7, 43.5, 38.7, 38.1, 25.7, 25.1, 24.9, 23.4, 23.0, 22.6, 21.4, 13.9, 13.7; $\mathrm{m} / \mathrm{z}(\mathrm{ESIMS}) 341.3(\mathrm{M}+23)^{+}$.

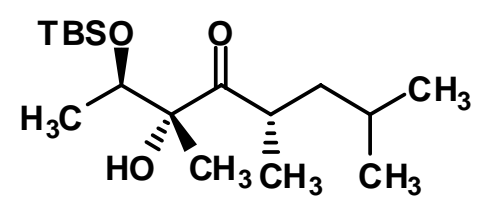

Allene 6c (40 mg, $0.134 \mathrm{mmol}$ ) was converted to the SDE using the general procedure. Procedure A was employed using $\mathrm{CuCN}(30 \mathrm{mg}, 0.335 \mathrm{mmol}), 1.6 \mathrm{M} \mathrm{MeLi}$ in $\mathrm{Et}_{2} \mathrm{O}(0.21 \mathrm{ml}$, $0.336 \mathrm{mmol})$ and $10 \mathrm{ml} \mathrm{Et}{ }_{2} \mathrm{O}$. FCC using $2 \%$ ethyl acetate-hexane gave $36 \mathrm{mg}(84 \%, 6: 1 \mathrm{dr})$ of $\alpha$-hydroxy ketone as a clear colorless oil $\left(\mathrm{R}_{\mathrm{f}}=0.60\right.$ in $10 \%$ ethyl acetate-hexanes). Spectral data for major isomer: IR $v_{\max }($ neat $) / \mathrm{cm}^{-1} 3555,1709,1464,838 ; \delta_{\mathrm{H}}\left(400 \mathrm{MHz}, \mathrm{CDCl}_{3}\right) 3.92(1 \mathrm{H}, \mathrm{q}$, $\mathrm{J}=6.0 \mathrm{~Hz}), 3.31-3.18(1 \mathrm{H}, \mathrm{m}), 2.92(1 \mathrm{H}, \mathrm{s}), 1.62-1.46(2 \mathrm{H}, \mathrm{m}), 1.28(1 \mathrm{H}, \mathrm{s}), 1.06-1.02(1 \mathrm{H}, \mathrm{m})$, 
$1.04(3 \mathrm{H}, \mathrm{d}, \mathrm{J}=6.4 \mathrm{~Hz}), 1.06(2.4 \mathrm{H}, \mathrm{d}, \mathrm{J}=6.4 \mathrm{~Hz}), 0.91(6 \mathrm{H}, \mathrm{d}, \mathrm{J}=10 \mathrm{~Hz}), 0.91(9 \mathrm{H}, \mathrm{s}), 0.10(3 \mathrm{H}$, s), $0.09(3 \mathrm{H}, \mathrm{s}) ; \delta_{\mathrm{c}}\left(100 \mathrm{MHz}, \mathrm{CDCl}_{3}\right) 218.5,82.3,73.4,42.1,38.8,25.8,25.6,24.3,23.2,21.9$, $18.5,18.0,15.7,-4.3,-4.9 ; \mathrm{m} / z\left(\right.$ ESIMS) $339.4(\mathrm{M}+23)^{+}$.

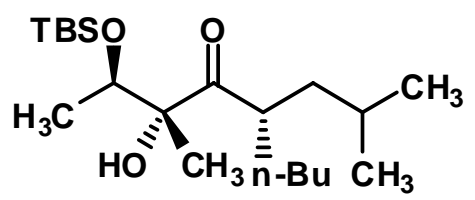

Allene 6c (32 mg, $0.119 \mathrm{mmol}$ ) was converted to the SDE using the general procedure. Procedure B was employed using CuCN (27 mg, $0.301 \mathrm{mmol}), 1.6 \mathrm{M} \mathrm{n}$-BuLi in hexane (0.18 ml, $0.288 \mathrm{mmol})$ and $8 \mathrm{ml} \mathrm{Et}_{2} \mathrm{O}$. FCC using $2 \%$ ethyl acetate-hexane gave $32.5 \mathrm{mg}(76 \%, 6: 1 \mathrm{dr})$ of $\alpha$-hydroxy ketone as a clear colorless oil $\left(R_{\mathrm{f}}=0.70\right.$ in $10 \%$ ethyl acetate-hexanes). Spectral data for major isomer: IR $v_{\max }$ (neat) $/ \mathrm{cm}^{-1} 3556,1706,1464,838 ; \delta_{\mathrm{H}}\left(400 \mathrm{MHz}, \mathrm{CDCl}_{3}\right) 3.94(1 \mathrm{H}$, q, J= $6 \mathrm{~Hz}), 3.19-3.10(1 \mathrm{H}, \mathrm{m}), 2.87(1 \mathrm{H}, \mathrm{s}), 1.68-1.48(3 \mathrm{H}, \mathrm{m}), 1.31-1.04(6 \mathrm{H}, \mathrm{m}), 1.25(3 \mathrm{H}, \mathrm{s})$, $1.05(3 \mathrm{H}, \mathrm{d}, \mathrm{J}=6.4 \mathrm{~Hz}), 0.89(9 \mathrm{H}, \mathrm{d}, \mathrm{J}=6.0 \mathrm{~Hz}), 0.92-0.82(9 \mathrm{H}, \mathrm{m}), 0.08(6 \mathrm{H}, \mathrm{s}) ; \delta_{\mathrm{c}}(100 \mathrm{MHz}$, $\left.\mathrm{CDCl}_{3}\right)$ 217.5, 82.2, 73.3, 44.4, 40.1, 29.9, 29.7, 25.9, 25.8, 24.8, 22.9(2), 22.5, 18.5, 18.0, 13.9, 4.2, -4.9; $\mathrm{m} / \mathrm{z}$ (ESIMS) $381.5(\mathrm{M}+23)^{+}$.

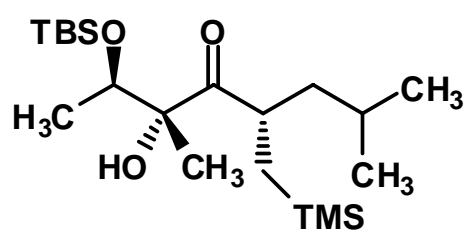

Allene 6c $(27 \mathrm{mg}, 0.301 \mathrm{mmol})$ was converted to the SDE using the general procedure. Procedure $\mathrm{C}$ was employed using $\mathrm{CuCN}(27 \mathrm{mg}, 0.301 \mathrm{mmol}), 1.0 \mathrm{M} \mathrm{TMSCH}_{2} \mathrm{Li}$ in pentane $(0.3$ $\mathrm{ml}, 0.30 \mathrm{mmol})$ and $8 \mathrm{ml} \mathrm{Et}{ }_{2} \mathrm{O}$. FCC using $2 \%$ ethyl acetate-hexane gave $31.5 \mathrm{mg}(68 \%, 6: 1 \mathrm{dr})$ of $\alpha$-hydroxy ketone as a clear colorless oil $\left(\mathrm{R}_{\mathrm{f}}=0.75\right.$ in $10 \%$ ethyl acetate-hexanes $)$. Spectral 
data for major isomer: IR $v_{\max }($ neat $) / \mathrm{cm}^{-1} 3557,1707,1464,1250 ; \delta_{\mathrm{H}}\left(400 \mathrm{MHz}, \mathrm{CDCl}_{3}\right) 4.03$ $(1 \mathrm{H}, \mathrm{q}, \mathrm{J}=6.4 \mathrm{~Hz}), 3.31-3.21(1 \mathrm{H}, \mathrm{m}), 2.66(1 \mathrm{H}, \mathrm{s}), 1.82-1.72(1 \mathrm{H}, \mathrm{m}), 1.41-1.28(1 \mathrm{H}, \mathrm{m}), 1.23$ $(3 \mathrm{H}, \mathrm{s}), 1.07(3 \mathrm{H}, \mathrm{d}, \mathrm{J}=6.4 \mathrm{~Hz}), 1.05-0.90(2 \mathrm{H}, \mathrm{m}), 0.91(9 \mathrm{H}, \mathrm{s}), 0.90(3 \mathrm{H}, \mathrm{d}, \mathrm{J}=6.4 \mathrm{~Hz}), 0.88$ $(3 \mathrm{H}, \mathrm{d}, \mathrm{J}=5.6 \mathrm{~Hz}), 0.38-0.30(1 \mathrm{H}, \mathrm{m}), 0.1(6 \mathrm{H}, \mathrm{s}), 0.05(9 \mathrm{H}, \mathrm{s}) ; \delta_{\mathrm{c}}\left(100 \mathrm{MHz}, \mathrm{CDCl}_{3}\right) 217.0$, 82.1, 72.7, 41.6, 39.6, 26.4, 25.8, 24.9, 23.7, 21.9, 18.7, 18.6, 18.0, -0.5, -4.2, -4.9; m/z (ESIMS) $411.4(\mathrm{M}+23)^{+}$.

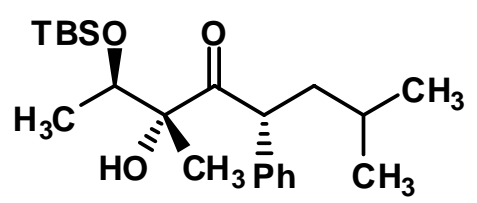

Allene 6c (50 mg, $0.186 \mathrm{mmol}$ ) was converted to the SDE using the general procedure. Procedure E was employed using CuI (107 mg, 0.562 mmol), 2.0 M PhLi in dibutylether (0.55 $\mathrm{ml}, 1.10 \mathrm{mmol})$ and $15 \mathrm{ml} \mathrm{Et}_{2} \mathrm{O}$. FCC using 2\% ethyl acetate-hexane gave 46mg (65 \%, 6:1 dr) of $\alpha$-hydroxy ketone as a clear colorless oil $\left(\mathrm{R}_{\mathrm{f}}=0.70\right.$ in $10 \%$ ethyl acetate-hexanes $)$. Spectral data for major isomer: IR $v_{\max }$ (neat) $/ \mathrm{cm}^{-1} 3583,1709,1257,1108,838 ; \delta_{\mathrm{H}}\left(400 \mathrm{MHz}, \mathrm{CDCl}_{3}\right)$ 7.31-7.24 (4H, m), 7.22-7.17 (1H, m), $4.50(1 \mathrm{H}, \mathrm{t}, \mathrm{J}=7.6 \mathrm{~Hz}), 2.90(1 \mathrm{H}, \mathrm{s}), 1.86-1.68(1 \mathrm{H}, \mathrm{m})$, 1.63-1.72 (1H, m), 1.36-1.28 (1H, m), $1.25(3 \mathrm{H}, \mathrm{s}), 0.90(3 \mathrm{H}, \mathrm{d}, \mathrm{J}=6.4 \mathrm{~Hz}), 0.87(3 \mathrm{H}, \mathrm{d}, \mathrm{J}=6.4$ $\mathrm{Hz}), 0.88(9 \mathrm{H}, \mathrm{s}), 0.73(3 \mathrm{H}, \mathrm{d}, \mathrm{J}=6.0 \mathrm{~Hz}), 0.05(3 \mathrm{H}, \mathrm{s}), 0.03(3 \mathrm{H}, \mathrm{s}) ; \delta_{\mathrm{c}}\left(100 \mathrm{MHz}, \mathrm{CDCl}_{3}\right)$ $214.2,138.2,129.1,128.3,126.8,82.7,72.9,50.6,42.6,25.8,25.5,24.6,23.0,22.0,18.4,18.0$, $-4.3,-4.9 ; m / z$ (ESIMS) $379.1(\mathrm{M}+23)^{+}$.

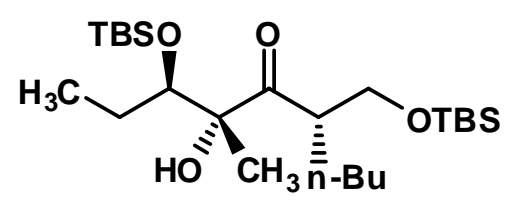


Allene 6d (50 mg, $0.135 \mathrm{mmol})$ was converted to the SDE using the general procedure. In a separate flask, a suspension of $\mathrm{CuCN}(60 \mathrm{mg}, 0.674 \mathrm{mmol})$ (activated by a gentle flame under vacuum) in $5 \mathrm{ml}$ of $\mathrm{Et}_{2} \mathrm{O}$ was degassed for $5 \mathrm{~min}$ using argon. The suspension was then cooled to $-78^{\circ} \mathrm{C}$ and a $1.6 \mathrm{M}$ solution of $\mathrm{n}-\mathrm{BuLi}$ in hexanes $(0.42 \mathrm{ml}, 0.674 \mathrm{mmol})$ was added dropwise. The suspension was warmed to $-20^{\circ} \mathrm{C}$ at which point a homogeneous solution was formed. The solution was then cooled to $-78^{\circ} \mathrm{C}$ and the above SDE in $1 \mathrm{ml}$ of $\mathrm{Et}_{2} \mathrm{O}$ was added dropwise. The reaction was slowly allowed to warm to $\mathrm{rt}$ over $1 \mathrm{hr}$ and stirred at $\mathrm{rt}$ for $6 \mathrm{~h}$. Upon completion of the reaction by tlc, saturated aq. $\mathrm{NH}_{4} \mathrm{Cl}(2 \mathrm{ml})$ was added. The $\mathrm{Et}_{2} \mathrm{O}$ layer was separated and washed again with saturated aq. $\mathrm{NH}_{4} \mathrm{Cl}(2 \times 5 \mathrm{ml})$. The aqueous layers were combined and extracted with $\mathrm{Et}_{2} \mathrm{O}(3 \times 10 \mathrm{ml})$. The $\mathrm{Et}_{2} \mathrm{O}$ layers were combined, dried using $\mathrm{MgSO}_{4}$ and evaporated. FCC using 1\% ethyl acetate-hexanes gave $41 \mathrm{mg}(66 \%, 8: 1 \mathrm{dr})$ of $\alpha$-hydroxy ketone as a clear colorless oil $\left(\mathrm{R}_{\mathrm{f}}=0.80\right.$ in $10 \%$ ethyl acetate-hexanes). Spectral data for major isomer: IR $v_{\max }$ (neat) $/ \mathrm{cm}^{-1} 3444,2949,2929,2851,1711,1474,1254,1106,841 ; \delta_{\mathrm{H}}(500 \mathrm{MHz}$, $\left.\mathrm{CDCl}_{3}\right)$ 3.89-3.66 (4H, m), 3.48-3.43 (1H, m), 1.59-1.22 (6H, m), $1.24(3 \mathrm{H}, \mathrm{s}), 0.94-0.86(26 \mathrm{H}$, m), $0.14(3 \mathrm{H}, \mathrm{s}), 0.11(3 \mathrm{H}, \mathrm{s}), 0.06(3 \mathrm{H}, \mathrm{s}), 0.04(3 \mathrm{H}, \mathrm{s}) ;\left(125 \mathrm{MHz}, \mathrm{CDCl}_{3}\right)$ 216.9, 82.6, 79.5, $65.0,49.2,29.6,27.7,26.2,25.9,25.8,24.3,23.0,18.4,18.3,13.9,11.3,-3.7,-4.2,-5.5,-5.6 ; \mathrm{m} / \mathrm{z}$ (ESIMS) $461.0(\mathrm{M}+1)^{+}$

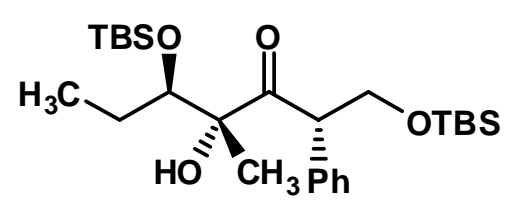

Allene 6d (30 mg, $0.081 \mathrm{mmol})$ was converted to the SDE using the general procedure. In a separate flask, a suspension of CuI (104 $\mathrm{mg}, 0.547 \mathrm{mmol})$ (activated by a gentle flame under vacuum) in $3 \mathrm{ml}$ of $\mathrm{Et}_{2} \mathrm{O}$ was degassed for $5 \mathrm{~min}$ using argon. The suspension was then cooled 
to $-78^{\circ} \mathrm{C}$ and a $2.0 \mathrm{M}$ solution of $\mathrm{PhLi}$ in dibutylether $(0.54 \mathrm{ml}, 1.08 \mathrm{mmol})$ of was added dropwise. The suspension was warmed to $0^{\circ} \mathrm{C}$ at which point the reaction mixture turned dark and a black precipitate was formed. This heterogeneous mixture was stirred for $5 \mathrm{~min}$ at $0^{\circ} \mathrm{C}$ and was then cooled to $-78^{\circ} \mathrm{C}$. The above spirodiepoxide in $1 \mathrm{ml}$ of $\mathrm{Et}_{2} \mathrm{O}$ was added dropwise at $78^{\circ} \mathrm{C}$. The reaction was slowly allowed to warm to rt over $3 \mathrm{hr}$ and stirred at $\mathrm{rt}$ for $2 \mathrm{~h}$. Upon completion of the reaction by tlc, saturated aq. $\mathrm{NH}_{4} \mathrm{Cl}(2 \mathrm{ml})$ was added. The $\mathrm{Et}_{2} \mathrm{O}$ layer was separated and washed again with saturated aq. $\mathrm{NH}_{4} \mathrm{Cl}(2 \times 2 \mathrm{ml})$. The aqueous layers were combined and extracted with $\mathrm{Et}_{2} \mathrm{O}(3 \times 5 \mathrm{ml})$. The $\mathrm{Et}_{2} \mathrm{O}$ layers were combined, dried using $\mathrm{MgSO}_{4}$ and evaporated. FCC using 5\% EtOAc/Hexanes gave $24 \mathrm{mg}(62 \%, 8: 1 \mathrm{dr})$ of $\alpha$-hydroxy ketone as a clear colorless oil $\left(\mathrm{R}_{\mathrm{f}}=0.75\right.$ in $10 \%$ ethyl acetate-hexanes $)$. Spectral data for major isomer: IR $v_{\max }$ (neat) $/ \mathrm{cm}^{-1} 3497,2953,2925,2855,1716,1470,1258,1095,833,776 ; \delta_{\mathrm{H}}(400$ $\left.\mathrm{MHz}, \mathrm{CDCl}_{3}\right) 7.29-7.19(5 \mathrm{H}, \mathrm{m}), 4.66(1 \mathrm{H}, \mathrm{dd}, \mathrm{J}=9.6,4.8 \mathrm{~Hz}), 4.19(1 \mathrm{H}, \mathrm{t}, \mathrm{J}=9.6 \mathrm{~Hz}), 3.79(1 \mathrm{H}$, dd, J=6.0, $1.6 \mathrm{~Hz}), 3.65(1 \mathrm{H}, \mathrm{dd}, \mathrm{J}=9.4,4.4 \mathrm{~Hz}), 3.02(1 \mathrm{H}, \mathrm{s}), 1.24-1.16(2 \mathrm{H}, \mathrm{m}), 1.21(3 \mathrm{H}, \mathrm{s})$, $0.86(9 \mathrm{H}, \mathrm{s}), 0.84(9 \mathrm{H}, \mathrm{s}), 0.63(3 \mathrm{H}, \mathrm{t}, \mathrm{J}=7.6 \mathrm{~Hz}), 0.05(6 \mathrm{H}, \mathrm{s}),-0.03(3 \mathrm{H}, \mathrm{s}),-0.01(3 \mathrm{H}, \mathrm{s}) ;(125$ $\left.\mathrm{MHz}, \mathrm{CDCl}_{3}\right)$ 212.3, 135.3, 128.9, 128.5, 127.3, 83.1, 77.7, 65.8, 55.2, 26.0, 25.9, 25.8, 24.6, $18.3(2), 10.5,-3.9,-4.1,-5.5,-5.6 ; \mathrm{m} / z$ (ESIMS) $503.4(\mathrm{M}+23)^{+}$

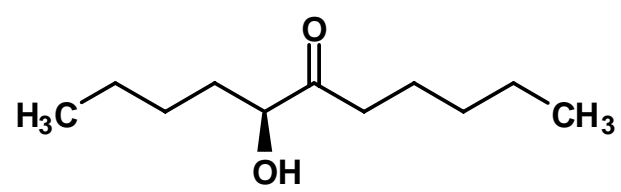

Allene 6a $(25 \mathrm{mg}, 0.140 \mathrm{mmol})$ was converted to the SDE using the general procedure. Procedure F was employed using $\mathrm{CuCN}(27 \mathrm{mg}, 0.30 \mathrm{mmol}), 1.0 \mathrm{M} \mathrm{MeMgBr}$ in dibutylether 
$(0.30 \mathrm{ml}, 0.30 \mathrm{mmol})$ and $3 \mathrm{ml} \mathrm{Et}{ }_{2} \mathrm{O}$. FCC using $2 \%$ ethyl acetate-hexane gave $18 \mathrm{mg}(72 \%)$ of known $\alpha$-hydroxy ketone ${ }^{8}$ as a clear colorless oil ( $\mathrm{R}_{\mathrm{f}}=0.50$ in $10 \%$ ethyl acetate-hexanes).

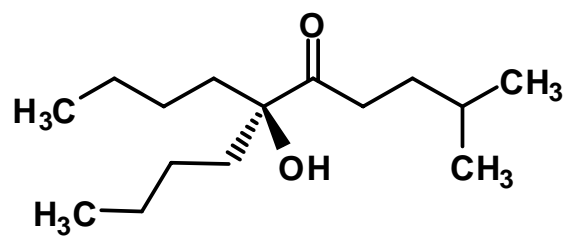

Allene 6a $(50 \mathrm{mg}, 0.210 \mathrm{mmol})$ was converted to the SDE using the general procedure. Procedure F was employed using $\mathrm{CuCN}$ (39 mg, $0.44 \mathrm{mmol}$ ), 1.0 M MeMgBr in dibutylether (0.44 ml, $0.44 \mathrm{mmol})$ and $5 \mathrm{ml} \mathrm{Et} 2 \mathrm{O}$. FCC using 2\% ethyl acetate-hexane gave $39 \mathrm{mg}(78 \%)$ of $\alpha$-hydroxy ketone as a clear colorless oil $\left(R_{\mathrm{f}}=0.55\right.$ in $10 \%$ ethyl acetate-hexanes). IR $v_{\max }$ (neat) $/ \mathrm{cm}^{-1} 3481.7,1703.9,1467.5 ; \delta_{\mathrm{H}}\left(400 \mathrm{MHz}, \mathrm{CDCl}_{3}\right) 3.903(1 \mathrm{H}, \mathrm{s}), 2.442(2 \mathrm{H}, \mathrm{t}, \mathrm{J}=7.6$ Hz), 1.76-1.65 (4H, m), 1.60-1.46 (4H, m), 1.44-1.23 (7H, m), $0.912(6 \mathrm{H}, \mathrm{d}, \mathrm{J}=6.4 \mathrm{~Hz}), 0.873$ $(6 \mathrm{H}, \mathrm{t}, \mathrm{J}=7.2 \mathrm{~Hz}) ; \delta_{\mathrm{c}}\left(100 \mathrm{MHz}, \mathrm{CDCl}_{3}\right) 214.8,81.6,38.7,33.8,32.3,27.6,25.4,22.9,22.3$, $13.9 ; \mathrm{m} / z$ (ESIMS) $243.0(\mathrm{M}+1)^{+}$.

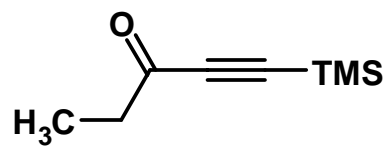

$14 a$

To a suspension of $\mathrm{AlCl}_{3}(10.09 \mathrm{~g}, 76.43 \mathrm{mmol})$ in $\mathrm{CH}_{2} \mathrm{Cl}_{2}(200 \mathrm{ml})$ was added a mixture of bis[trimethylsilyl]acetylene $(10.00 \mathrm{~g}, 58.82 \mathrm{mmol})$ and propionyl chloride $(5.11 \mathrm{ml}, 58.82$ mmol $)$ in $\mathrm{CH}_{2} \mathrm{Cl}_{2}(10 \mathrm{ml})$ dropwise at $-10^{\circ} \mathrm{C}$. The reaction was allowed to warm to $\mathrm{rt}$ and stirred for an additional $1 \mathrm{~h}$. After cooling to $-78^{\circ} \mathrm{C}, 1 \mathrm{~N} \mathrm{HCl}(50 \mathrm{ml})$ was added dropwise and the reaction was allowed to warm to rt. The $\mathrm{CH}_{2} \mathrm{Cl}_{2}$ layer was separated and the aqueous layer was

\footnotetext{
${ }^{8}$ Hashiyama, T.; Morikawa, K.; Sharpless, K.B. J. Org. Chem.1992, 57, 5067-5068.
} 
extracted with $\mathrm{CH}_{2} \mathrm{Cl}_{2}(3 \times 50 \mathrm{ml})$. The organic layers were combined, dried using $\mathrm{Na}_{2} \mathrm{SO}_{4}$, and evaporated to give $8.60 \mathrm{~g}$ of known alkynone ${ }^{9}(95 \%)$ as a yellow oil. This material was used for the next step without further purification.

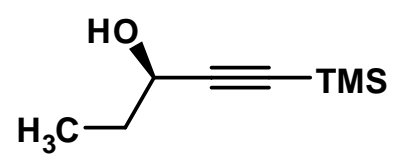

$14 b$

In a glove bag, $50 \mathrm{mg}$ of dichloro(p-cymene)ruthenium (II) dimer (0.082 mmol), $60 \mathrm{mg}$ of (1R,2R)-(+)-N-p-tosyl-1,2-diphenylethylenediamine (0.164 mmol), and $65 \mathrm{mg}$ of potassium hydroxide $(1.16 \mathrm{mmol})$ were added to a flask followed by addition of $2 \mathrm{~mL}$ of anhydrous $\mathrm{CH}_{2} \mathrm{Cl}_{2}$. The orange colored reaction was removed from the glove bag and stirred for $5 \mathrm{~min}$. after which time a purple colored appeared (Note: On some occasions the reaction turned purple immediately upon addition of $\mathrm{CH}_{2} \mathrm{Cl}_{2}$ ). $2 \mathrm{~mL}$ of water and $2 \mathrm{~mL}$ of $\mathrm{CH}_{2} \mathrm{Cl}_{2}$ were added to the reaction. The $\mathrm{CH}_{2} \mathrm{Cl}_{2}$ layer was separated and washed again with $2 \mathrm{~mL}$ of water. $\mathrm{The}^{\mathrm{CH}} \mathrm{Cl}_{2}$ layer was dried using $\mathrm{CaH}$, filtered, and evaporated to give $100 \mathrm{mg}$ of the Noyori catalyst as dark purple crystals. To this catalyst was added $25 \mathrm{~mL}$ of anhydrous isopropanol at $\mathrm{rt}$. Upon complete dissolution of the catalyst the solution was dark orange in color. 14a in $8 \mathrm{~mL}$ of anhydrous isopropanol was added in $0.5 \mathrm{~mL}$ portions based on the color change in the reaction. Upon addition of one portion of the alkynone the reaction immediately went from dark orange to dark purple and back to dark orange. At this time another portion of alkynone in isopropanol was added. This process was repeated until all alkynone was added (approximately $20 \mathrm{~min}$.). Upon completion of the reaction by tlc, the isopropanol was evaporated to give a dark brown crude oil. FCC using $10 \%$ ethyl acetate-hexanes gave $1.93 \mathrm{~g}$ of known propargyl alcohol ${ }^{9} \mathbf{1 4 b}$

\footnotetext{
${ }^{9}$ Denmark, S. E.; Yang, S. J. Am. Chem. Soc. 2004, 126, 12432.
} 
$(90 \%)$ as a pale yellow oil $\left(\mathrm{R}_{\mathrm{f}}=0.20\right.$ in $10 \%$ ethyl acetate-hexanes). Mosher ester analysis of the alcohol showed a single isomer indicating $>95: 5$ ee.

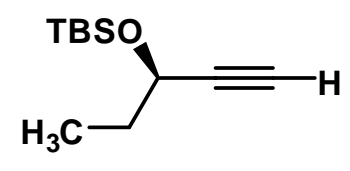

14

To a solution of $\mathbf{1 4 b}(3.70 \mathrm{~g}, 23.70 \mathrm{mmol})$ in $100 \mathrm{ml} \mathrm{CH}_{2} \mathrm{Cl}_{2}$ was added imidazole ( 2.23 $\mathrm{g}, 32.70 \mathrm{mmol})$, DMAP (133 $\mathrm{mg}, 1.08 \mathrm{mmol})$ and TBSCl (3.65 g, $24.17 \mathrm{mmol})$ at rt. The reaction stirred at $\mathrm{rt}$ and was monitored by tlc. Upon completion of the reaction by tlc, $100 \mathrm{ml}$ of a sat. solution of $\mathrm{K}_{2} \mathrm{CO}_{3}$ in $\mathrm{MeOH}$ was added. The reaction stirred for $2 \mathrm{~h}$ at $\mathrm{rt}$ followed by addition of $50 \mathrm{ml}$ of water. The $\mathrm{CH}_{2} \mathrm{Cl}_{2}$ layer was separated and the aqueous layer was extracted with $\mathrm{CH}_{2} \mathrm{Cl}_{2}(2 \times 50 \mathrm{ml})$. The organic layers were combined and dried using $\mathrm{NaSO}_{4}$. The solvent was slowly removed by rotovap in order to avoid loss of the volatile product. FCC using pentane gave $2.35 \mathrm{~g}$ of TBS-protected propargyl alcohol $14(50 \%)$ as a clear colorless oil $\left(\mathrm{R}_{\mathrm{f}}=\right.$ 0.80 in pentane). Enantiomer of $\mathbf{1 4}$ is a known compound. See: Xu, L.; Wu, X.; Zheng, G.R.; Cai, J.C. Chinese Chem. Lett. 2000, 11, 213-216. $[\alpha]_{\mathrm{D}}+41.5(c=0.020, \mathrm{MeOH})$. IR $v_{\max }$ (neat) / $\mathrm{cm}^{-1} 2962,2929,2859,1471,1249,1082,837 ; \delta \mathrm{H}\left(400 \mathrm{MHz}, \mathrm{CDCl}_{3}\right) 4.28(1 \mathrm{H}, \mathrm{td}, \mathrm{J}=6.4,2.0$ $\mathrm{Hz}), 2.37(1 \mathrm{H}, \mathrm{d}, \mathrm{J}=2.0 \mathrm{~Hz}), 1.74-1.65(2 \mathrm{H}, \mathrm{m}), 0.98(3 \mathrm{H}, \mathrm{t}, \mathrm{J}=7.2 \mathrm{~Hz}), 0.91(9 \mathrm{H}, \mathrm{s}), 0.14(3 \mathrm{H}$, s), $0.11(3 \mathrm{H}, \mathrm{s}) ; \delta \mathrm{c}\left(100 \mathrm{MHz}, \mathrm{CDCl}_{3}\right) \quad 85.5,71.9,64.0,31.7,25.8,18.2,9.5,-4.6,-5.1 ; \mathrm{m} / \mathrm{z}$ (ESIMS) $221.4(\mathrm{M}+23)^{+}$.

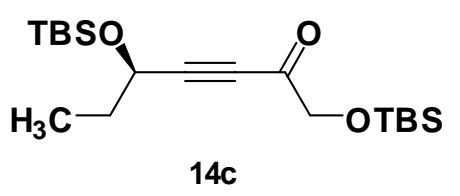


To a solution of $2.39 \mathrm{~g}$ of $\mathbf{1 4}(12.070 \mathrm{mmol})$ in $40 \mathrm{~mL}$ of anhydrous $\mathrm{Et}_{2} \mathrm{O}$ was added 6.86 $\mathrm{mL}$ of $1.6 \mathrm{M} \mathrm{n}-\mathrm{BuLi}(10.976 \mathrm{mmol})$ at $-78^{\circ} \mathrm{C}$. The reaction stirred at $-78^{\circ} \mathrm{C}$ for $20 \mathrm{~min}$. and was allowed to warm to rt. Reaction stirred at rt for $30 \mathrm{~min}$. before cooling back down to $-78^{\circ} \mathrm{C}$. $2.34 \mathrm{~g}$ of known weinreb amide $\mathbf{1 3}^{10}(10.043 \mathrm{mmol})$ in $10 \mathrm{~mL}$ of anhydrous $\mathrm{Et}_{2} \mathrm{O}$ was added dropwise at $-78^{\circ} \mathrm{C}$. The reaction was placed in a $-20^{\circ} \mathrm{C}$ freezer overnight. $25 \mathrm{~mL}$ of saturated aq. $\mathrm{NH}_{4} \mathrm{Cl}$ was added to the reaction at $-20^{\circ} \mathrm{C}$ and was let to warm to rt. $\mathrm{The}_{2} \mathrm{O}$ layer was separated and the aqueous layer was extracted twice using $10 \mathrm{~mL}$ of $\mathrm{Et}_{2} \mathrm{O}$. $\mathrm{The}_{2} \mathrm{O}$ layers were combined, dried using $\mathrm{MgSO}_{4}$, and evaporated to give alkynone $\mathbf{1 4} \mathbf{c}$ as a pale yellow oil. This crude material was taken onto the next step without further purification $\left(\mathrm{R}_{\mathrm{f}}=0.65\right.$ in $5 \%$ ethyl acetate-hexanes). $[\alpha]_{\mathrm{D}}+24.5(c=0.011, \mathrm{MeOH}) . \mathrm{IR} v_{\max }$ (neat) $/ \mathrm{cm}^{-1} 2953,2952,2859,2210$, $1703,1679,1462,1254,1143,1111 ; \delta \mathrm{H}\left(400 \mathrm{MHz}, \mathrm{CDCl}_{3}\right) 4.44(1 \mathrm{H}, \mathrm{t}, \mathrm{J}=6.4 \mathrm{~Hz}), 4.34(2 \mathrm{H}, \mathrm{s})$, 1.78-1.71 (2H, m), $0.99(3 \mathrm{H}, \mathrm{t}, \mathrm{J}=7.2 \mathrm{~Hz}), 0.92(9 \mathrm{H}, \mathrm{s}), 0.91(9 \mathrm{H}, \mathrm{s}), 0.14(3 \mathrm{H}, \mathrm{s}), 0.11(3 \mathrm{H}, \mathrm{s})$, $0.10(6 \mathrm{H}, \mathrm{s}) ; \delta \mathrm{c}\left(100 \mathrm{MHz}, \mathrm{CDCl}_{3}\right)$ 186.0, 96.0, 80.9, 70.5, 64.0, 31.0, 25.8, 25.7, 18.4, 18.2, 9.5, -4.6, -5.1, -5.4 (2); $\mathrm{m} / \mathrm{z}$ (ESIMS) $371.1(\mathrm{M}+1)^{+}$.

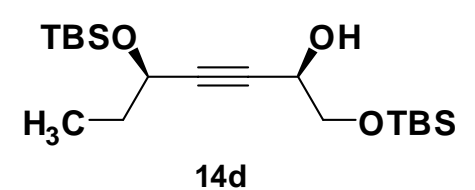

In a glove bag, $150 \mathrm{mg}$ of dichloro(p-cymene)ruthenium (II) dimer (0.245 mmol), 180 $\mathrm{mg}$ of (1S,2S)-(+)-N-p-tosyl-1,2-diphenylethylenediamine (0.492 mmol), and $195 \mathrm{mg}$ of potassium hydroxide $(3.480 \mathrm{mmol})$ were added to a flask followed by addition of $4 \mathrm{~mL}$ of anhydrous $\mathrm{CH}_{2} \mathrm{Cl}_{2}$. The orange colored reaction was removed from the glove bag and stirred for

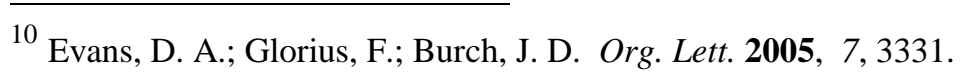


5 min. after which time a purple colored appeared. (Note: On some occasions the reaction turned purple immediately upon addition of $\left.\mathrm{CH}_{2} \mathrm{Cl}_{2}\right) 5 \mathrm{~mL}$ of water and $5 \mathrm{~mL}$ of $\mathrm{CH}_{2} \mathrm{Cl}_{2}$ were added to the reaction. The $\mathrm{CH}_{2} \mathrm{Cl}_{2}$ layer was separated and washed again with $5 \mathrm{~mL}$ of water. The $\mathrm{CH}_{2} \mathrm{Cl}_{2}$ layer was dried using $\mathrm{CaH}$, filtered, and evaporated to give $300 \mathrm{mg}(0.500 \mathrm{mmol})$ of the Noyori catalyst as dark purple crystals. To this catalyst was added $60 \mathrm{~mL}$ of anhydrous isopropanol at rt. Upon complete dissolution of the catalyst the solution was dark orange in color. $14 \mathbf{c}$ in $10 \mathrm{~mL}$ of anhydrous isopropanol was added in $1 \mathrm{~mL}$ portions based on the color change in the reaction. Upon addition of one portion of the alkynone the reaction immediately went from dark orange to dark purple and back to dark orange. At this time another portion of alkynone in isopropanol was added. This process was repeated until all alkynone was added (approximately 20 min.). Upon completion of the reaction by tlc, the isopropanol was evaporated to give a dark brown crude oil. FCC using first hexanes than 3\% ethyl acetatehexanes gave $3.25 \mathrm{~g}$ of propargyl alcohol $\mathbf{1 4 d}(87 \%$ over 2 steps, $>95: 5 \mathrm{dr})$ as a pale yellow oil $\left(\mathrm{R}_{\mathrm{f}}=0.50\right.$ in $5 \%$ ethyl acetate-hexanes). $[\alpha]_{\mathrm{D}}+25.2(c=0.088, \mathrm{MeOH}) . \mathrm{IR} v_{\max }$ (neat) $/ \mathrm{cm}^{-1}$ $3374,2953,2929,2855,2218,1719,1679,1454,1254,1082 ; \delta_{\mathrm{H}}\left(400 \mathrm{MHz}, \mathrm{CDCl}_{3}\right) 4.45-4.40$ $(1 \mathrm{H}, \mathrm{m}), 4.31(1 \mathrm{H}, \mathrm{td}, \mathrm{J}=6.4,1.6 \mathrm{~Hz}), 3.75(1 \mathrm{H}, \mathrm{dd}, \mathrm{J}=10.0,4.0 \mathrm{~Hz}), 3.62(1 \mathrm{H}, \mathrm{dd}, \mathrm{J}=8.4,7.6 \mathrm{~Hz})$, $2.51(1 \mathrm{H}, \mathrm{br}), 1.70-1.63(2 \mathrm{H}, \mathrm{m}), 0.96(3 \mathrm{H}, \mathrm{t}, \mathrm{J}=7.2 \mathrm{~Hz}), 0.91(9 \mathrm{H}, \mathrm{s}), 0.90(9 \mathrm{H}, \mathrm{s}), 0.12(3 \mathrm{H}, \mathrm{s})$, $0.10(3 \mathrm{H}, \mathrm{s}), 0.09$ (6H, s); $\delta \mathrm{c}\left(100 \mathrm{MHz}, \mathrm{CDCl}_{3}\right)$ 87.1, 81.7, 67.0, 64.2, 63.1, 31.7, $25.8(2)$, $18.3,18.2,9.7,-4.5,-4.9,-5.3,-5.4 ; \mathrm{m} / z$ (ESIMS) $397.8(\mathrm{M}+23)^{+}$.

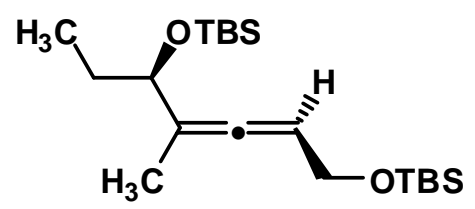

$6 d$ 
To a solution of $1.21 \mathrm{~g}$ ( $3.253 \mathrm{mmol})$ of $\mathbf{1 4 b}$ and $0.59 \mathrm{~mL}$ (4.240 mmol) of triethylamine in $50 \mathrm{~mL}$ of $\mathrm{CH}_{2} \mathrm{Cl}_{2}$ was added $0.30 \mathrm{~mL}$ (3.895 mmol) of methanesulfonyl chloride dropwise at $78^{\circ} \mathrm{C}$. The reaction was slowly allowed to warm to rt over $1 \mathrm{~h}$ and stirred for $30 \mathrm{~min}$. at rt. 25 $\mathrm{mL}$ of water was added and the $\mathrm{CH}_{2} \mathrm{Cl}_{2}$ layer was separated and washed again with $25 \mathrm{~mL}$ of water. The $\mathrm{CH}_{2} \mathrm{Cl}_{2}$ layer was separated, dried using $\mathrm{Na}_{2} \mathrm{SO}_{4}$ and evaporated. The crude pale yellow oil was used for the next step without purification.

In a separate flask, $579 \mathrm{mg}(6.465 \mathrm{mmol})$ of $\mathrm{CuCN}$ (activated by a gentle flame under high vacuum) in $40 \mathrm{~mL}$ of anhydrous $\mathrm{Et}_{2} \mathrm{O}$ was degassed for $10 \mathrm{~min}$ with argon. This suspension was cooled to $-20^{\circ} \mathrm{C}$ and $4.00 \mathrm{~mL}(6.400 \mathrm{mmol})$ of $1.6 \mathrm{M} \mathrm{MeLi}$ in ether was added dropwise. The suspension went from a bright canary yellow color to a homogeneous clear colorless solution. A solution of the above mesylate in $10 \mathrm{~mL}$ of anhydrous $\mathrm{Et}_{2} \mathrm{O}$ was added dropwise to the reaction at $-20^{\circ} \mathrm{C}$. The reaction immediately formed a bright canary yellow precipitate and was allowed to warm to rt over $1 \mathrm{~h}$. Upon completion by tlc, the reaction was quenched at $\mathrm{rt}$ using $30 \mathrm{~mL}$ of saturated aq. $\mathrm{NH}_{4} \mathrm{Cl}$. The ether layer was separated and washed again with 30 $\mathrm{mL}$ of saturated aq. $\mathrm{NH}_{4} \mathrm{Cl}$. The aqueous layers were combined and extracted using $\mathrm{Et}_{2} \mathrm{O}(2 \times 20$ $\mathrm{mL}$ ). The $\mathrm{Et}_{2} \mathrm{O}$ layers were combined, dried using $\mathrm{MgSO}_{4}$ and evaporated. $\mathrm{FCC}$ using $1 \%$ ether-pentane gave $1.18 \mathrm{~g}$ of allene $\mathbf{6 d}(98 \%,>95: 5 \mathrm{dr})$ as a colorless oil $\left(\mathrm{R}_{\mathrm{f}}=0.90\right.$ in 5\% ethyl acetate-hexanes). $[\alpha]_{\mathrm{D}}+20.8(c=0.024, \mathrm{MeOH}) . \mathrm{IR} v_{\max }$ (neat) $/ \mathrm{cm}^{-1} 2962,2917,2888,2859$, $1969,1470,1462,1254,1090 ; \delta_{\mathrm{H}}\left(400 \mathrm{MHz}, \mathrm{CDCl}_{3}\right)$ 5.17-5.12 $(1 \mathrm{H}, \mathrm{m}), 4.22-4.12(2 \mathrm{H}, \mathrm{m})$, $4.02(1 \mathrm{H}, \mathrm{t}, \mathrm{J}=6.8 \mathrm{~Hz}), 1.63(3 \mathrm{H}, \mathrm{d}, \mathrm{J}=2.8 \mathrm{~Hz}), 1.62-1.48(2 \mathrm{H}, \mathrm{m}), 0.91(9 \mathrm{H}, \mathrm{s}), 0.89(9 \mathrm{H}, \mathrm{s}), 0.84$ (3H, t, J=7.4 Hz), $0.08(6 \mathrm{H}, \mathrm{s}), 0.04(6 \mathrm{H}, \mathrm{s}) ; \delta \mathrm{c}\left(100 \mathrm{MHz}, \mathrm{CDCl}_{3}\right)$ 200.4, 102.9, 91.2, 75.7, 62.2, 29.1, 26.0, 25.9, 18.4, 18.2, 12.8, 10.2, -4.6, -5.0 (2), -5.1; $\mathrm{m} / \mathrm{z}$ (ESIMS) $393.4(\mathrm{M}+23)^{+}$. 


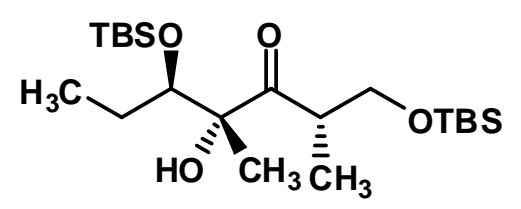

15

Allene 6d (506 mg, $1.367 \mathrm{mmol})$ was converted to the SDE using the general procedure. To $\mathrm{CuCN}$ (601 mg, $6.710 \mathrm{mmol}$ ) (activated by a gentle flame under high vacuum) was added $50 \mathrm{~mL}$ of anhydrous $\mathrm{Et}_{2} \mathrm{O}$. The suspension was degassed for 10 min using argon. The suspension was then cooled to $-78^{\circ} \mathrm{C}$ and $4.20 \mathrm{ml}(6.720 \mathrm{mmol})$ of $1.6 \mathrm{M} \mathrm{MeLi}$ in $\mathrm{Et}_{2} \mathrm{O}$ was added dropwise. The reaction was warmed to $0^{\circ} \mathrm{C}$ over $10 \mathrm{~min}$ and stirred at $0^{\circ} \mathrm{C}$ for $5 \mathrm{~min}$. The reaction had gone from heterogeneous to a clear pale yellow homogeneous solution at $0^{\circ} \mathrm{C}$. The reaction was then recooled to $-78^{\circ} \mathrm{C}$ and the above spirodiepoxide in $5 \mathrm{~mL}$ of anhydrous $\mathrm{Et}_{2} \mathrm{O}$ was added dropwise. A bright canary yellow precipitate immediately formed and the reaction was let to warm to rt over $1.5 \mathrm{~h}$ and stirred at $\mathrm{rt}$ for $4 \mathrm{~h}$. Upon completion of the reaction by tlc saturated aq. $\mathrm{NH}_{4} \mathrm{Cl}(20 \mathrm{ml})$ was added. The $\mathrm{Et}_{2} \mathrm{O}$ layer was separated and washed again with saturated aq. $\mathrm{NH}_{4} \mathrm{Cl}(2 \times 15 \mathrm{ml})$. The aqueous layers were combined and extracted with $\mathrm{Et}_{2} \mathrm{O}(3 \times 20 \mathrm{ml})$. The $\mathrm{Et}_{2} \mathrm{O}$ layers were combined, dried using $\mathrm{MgSO}_{4}$ and evaporated. $\mathrm{FCC}$ using $5 \%$ ethyl acetate-hexanes gave $456 \mathrm{mg}$ of $\alpha$-hydroxy ketone $15(80 \%, 8: 1 \mathrm{dr})$ as a clear colorless oil $\left(\mathrm{R}_{\mathrm{f}}=\right.$ 0.70 in $10 \%$ ethyl acetate-hexanes). Spectral data for major isomer: $\operatorname{IR} v_{\max }$ (neat) $/ \mathrm{cm}^{-1} 3469$, $2949,2856,1712,1467,1251,1100,843 ; \delta_{\mathrm{H}}\left(400 \mathrm{MHz}, \mathrm{CDCl}_{3}\right) 3.76(1 \mathrm{H}, \mathrm{s}), 3.68-3.57(4 \mathrm{H}, \mathrm{m})$, 1.58-1.36 (2H, m), $1.23(3 \mathrm{H}, \mathrm{s}), 0.97(3 \mathrm{H}, \mathrm{d}, \mathrm{J}=6.4 \mathrm{~Hz}), 0.93-0.86(21 \mathrm{H}, \mathrm{m}), 0.14(3 \mathrm{H}, \mathrm{s}), 0.09$ (3H, s), $0.06(3 \mathrm{H}, \mathrm{s}), 0.04(3 \mathrm{H}, \mathrm{s}) ; \delta \mathrm{c}\left(100 \mathrm{MHz}, \mathrm{CDCl}_{3}\right)$ 217.5, 82.5, 79.5, 66.7, 43.4, 29.7, $26.1,25.9,24.0,18.4,18.3,12.9,11.2,-3.7,-4.3,-5.5,-5.7 ; \mathrm{m} / z$ (ESIMS) $419.0(\mathrm{M}+1)^{+}$. 


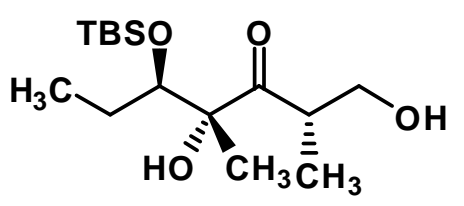

$15 a$

$97 \mathrm{mg}(0.232 \mathrm{mmol})$ of $15(8: 1 \mathrm{dr})$ in $2.5 \mathrm{~mL}$ of acetic acid, $0.9 \mathrm{~mL}$ of water, and $0.9 \mathrm{~mL}$ of THF was stirred at $\mathrm{rt}$ for $12 \mathrm{~h} .10 \mathrm{ml}$ of water and $15 \mathrm{ml} \mathrm{of} \mathrm{CH}_{2} \mathrm{Cl}_{2}$ were added. $\mathrm{The}^{\mathrm{CH}_{2} \mathrm{Cl}_{2}}$ layer was separated and washed again with $10 \mathrm{~mL}$ of water. The aqueous layers were combined, dried using $\mathrm{Na}_{2} \mathrm{SO}_{4}$ and evaporated. FCC using $15 \%$ ethyl acetate-hexane gave $60 \mathrm{mg}$ of diol $(8: 1 \mathrm{dr})$ as a clear colorless oil. At this stage the isomers were readily separable by FCC under the same conditions to give $\mathbf{1 5 a}$ as a single diastereomer $(85 \%)\left(\mathrm{R}_{\mathrm{f}}=0.30\right.$ in $20 \%$ ethyl acetatehexanes). Spectral data for 15a: $[\alpha]_{\mathrm{D}}+58.1(c=0.016, \mathrm{MeOH}) . \mathrm{IR} v_{\max }$ (neat) $/ \mathrm{cm}^{-1} 3423,2953$, 2929, 2888, 2855, 1707, 1462, 1254, 1123, 1017, 1008; $\delta_{\mathrm{H}}\left(400 \mathrm{MHz}, \mathrm{CDCl}_{3}\right) 3.76-3.64(3 \mathrm{H}$, m), 3.55-3.46 (1H, m), $3.39(1 \mathrm{H}, \mathrm{s}), 2.57(1 \mathrm{H}, \mathrm{br}), 1.60-1.49(2 \mathrm{H}, \mathrm{m}), 1.31(3 \mathrm{H}, \mathrm{s}), 1.05(3 \mathrm{H}, \mathrm{d}$, $\mathrm{J}=6.8 \mathrm{~Hz}), 0.93(3 \mathrm{H}, \mathrm{t}, \mathrm{J}=7.6 \mathrm{~Hz}), 0.92(9 \mathrm{H}, \mathrm{s}), 0.14(3 \mathrm{H}, \mathrm{s}), 0.12(3 \mathrm{H}, \mathrm{s}) ; \delta \mathrm{c}\left(100 \mathrm{MHz}, \mathrm{CDCl}_{3}\right)$ 218.0, 83.0, 79.6, 65.8, 43.7, 26.2, 26.0, 24.2, 18.3, 13.3, 11.2, -4.0, -4.1; $\mathrm{m} / \mathrm{z}$ (ESIMS) 305.0 $(\mathrm{M}+1)^{+}$

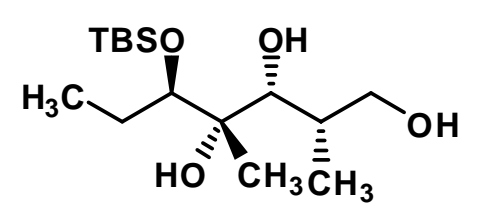

16

To a solution of $418 \mathrm{mg}(1.589 \mathrm{mmol})$ of tetramethylammonium triacetoxyborohydride in $0.8 \mathrm{~mL}$ of acetic acid and $1.3 \mathrm{~mL}$ of acetonitrile was added $100 \mathrm{mg}(0.329 \mathrm{mmol})$ of $\mathbf{1 5 a}$ at $40^{\circ} \mathrm{C}$. The reaction stirred at $-40^{\circ} \mathrm{C}$ for $12 \mathrm{~h}$ and was let to warm to rt. Upon completion of the reaction by TLC, $2 \mathrm{~mL}$ of saturated aq. $\mathrm{NaHCO}_{3}$ and $10 \mathrm{~mL}$ of $\mathrm{CH}_{2} \mathrm{Cl}_{2}$ were added. The $\mathrm{CH}_{2} \mathrm{Cl}_{2}$ 
layer was separated and washed again with $5 \mathrm{~mL}$ of sat. $\mathrm{NaHCO}_{3}$. The aqueous layers were combined and extracted with $\mathrm{CH}_{2} \mathrm{Cl}_{2}(2 \times 5 \mathrm{~mL})$. The $\mathrm{CH}_{2} \mathrm{Cl}_{2}$ layers were combined, dried using $\mathrm{Na}_{2} \mathrm{SO}_{4}$ and evaporated. FCC using 20\% ethyl acetate-hexane gave $91 \mathrm{mg}$ of the triol (90\%, 6:1 dr) as a clear colorless oil ( $\mathrm{R}_{\mathrm{f}}=0.50$ in $50 \%$ ethyl acetate-hexanes). Careful separation by FCC under the same conditions gave the major isomer 16. Spectral data for 16: $[\alpha]_{D}+13.5(c=0.023$, $\mathrm{MeOH})$. IR $v_{\max }$ (neat) / $\mathrm{cm}^{-1} 3403,2958,2925,2884,2859,1470,1462,1249,1098,1045,837$; $\delta_{\mathrm{H}}\left(300 \mathrm{MHz}, \mathrm{CDCl}_{3}\right) 4.11(1 \mathrm{H}, \mathrm{d}, \mathrm{J}=1.5 \mathrm{~Hz}), 3.74(1 \mathrm{H}, \mathrm{dd}, \mathrm{J}=10.6,3.9 \mathrm{~Hz}), 3.64(1 \mathrm{H}, \mathrm{dd}$, $\mathrm{J}=10.5,6.0 \mathrm{~Hz}), 3.50(1 \mathrm{H}, \mathrm{dd}, \mathrm{J}=7.8,3.3 \mathrm{~Hz}), 1.86-1.79(1 \mathrm{H}, \mathrm{m}), 1.69-1.42(2 \mathrm{H}, \mathrm{m}), 1.09(3 \mathrm{H}$, d, J=6.9 Hz), $1.07(3 \mathrm{H}, \mathrm{s}), 1.00(3 \mathrm{H}, \mathrm{t}, \mathrm{J}=7.5 \mathrm{~Hz}), 0.93(9 \mathrm{H}, \mathrm{s}), 0.18(3 \mathrm{H}, \mathrm{s}), 0.14(3 \mathrm{H}, \mathrm{s}) ;(75$ $\left.\mathrm{MHz}, \mathrm{CDCl}_{3}\right)$ 85.7, 74.8, 74.3, 68.3, 36.2, 26.5, 26.1, 21.0, 18.3, 12.1, 10.6, -4.0, -4.3; $\mathrm{m} / \mathrm{z}$ (ESIMS) $308.2(\mathrm{M}+1)^{+}$.

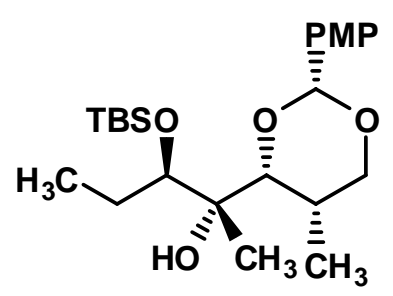

$16 a$

To a solution of $39 \mathrm{mg}(0.127 \mathrm{mmol})$ of $\mathbf{1 6}$ in $1 \mathrm{~mL}$ of $\mathrm{CH}_{2} \mathrm{Cl}_{2}$ was added $54 \mu \mathrm{L}(0.320$ mmol) of anisaldehyde dimethyl acetal and $1 \mathrm{mg}$ of pyridinium p-toluenesulfonate (PPTS) at rt. Reaction stirred for $30 \mathrm{~min}$. at rt. $2 \mathrm{~mL}$ of saturated aq. $\mathrm{NaHCO}_{3}$ and $10 \mathrm{~mL}$ of $\mathrm{CH}_{2} \mathrm{Cl}_{2}$ was added. The $\mathrm{CH}_{2} \mathrm{Cl}_{2}$ layer was separated, dried using $\mathrm{Na}_{2} \mathrm{SO}_{4}$ and evaporated. FCC using 5\% ethyl acetate-hexane gave $49 \mathrm{mg}$ of PMP acetal 16a $(91 \%)$ as a clear colorless oil $\left(\mathrm{R}_{\mathrm{f}}=0.40\right.$ in $10 \%$ ethyl acetate-hexanes) $\left(\mathrm{R}_{\mathrm{f}}=0.60\right.$ in $20 \%$ ethyl acetate-hexanes). $[\alpha]_{\mathrm{D}}-6.6(c=0.029$, MeOH). IR $v_{\max }$ (neat) $/ \mathrm{cm}^{-1} 3566,2953,2929,2851,1617,1519,1466,1380,1249,1111$, 
$1041 ; \delta_{\mathrm{H}}\left(400 \mathrm{MHz}, \mathrm{CDCl}_{3}\right) 7.42(2 \mathrm{H}, \mathrm{d}, \mathrm{J}=11.6 \mathrm{~Hz}), 6.92(2 \mathrm{H}, \mathrm{d}, \mathrm{J}=11.2 \mathrm{~Hz}), 5.58(1 \mathrm{H}, \mathrm{s}), 4.11$

$(1 \mathrm{H}, \mathrm{d}, \mathrm{J}=3.2 \mathrm{~Hz}), 4.06-3.97(2 \mathrm{H}, \mathrm{m}), 3.82(3 \mathrm{H}, \mathrm{s}), 3.62(1 \mathrm{H}, \mathrm{dd}, \mathrm{J}=8.0,6.0 \mathrm{~Hz}), 2.20(1 \mathrm{H}, \mathrm{br})$ 1.93-1.71 (2H, m), 1.45-1.30 (1H, m), $1.35(3 \mathrm{H}, \mathrm{d}, \mathrm{J}=9.2 \mathrm{~Hz}), 0.95(3 \mathrm{H}, \mathrm{t}, \mathrm{J}=10.0 \mathrm{~Hz}), 0.92(9 \mathrm{H}$, s), $0.11(3 \mathrm{H}, \mathrm{s}), 0.09(3 \mathrm{H}, \mathrm{s}) ;\left(100 \mathrm{MHz}, \mathrm{CDCl}_{3}\right)$ 160.0, 131.2, 127.4, 113.6, 101.7, 78.6, 76.5, $75.8,75.1,55.3,30.3,26.1,24.8,18.2,14.7,13.0,12.3,-3.3,-4.4 ; \mathrm{m} / z$ (ESIMS) $449.0(\mathrm{M}+23)^{+}$.

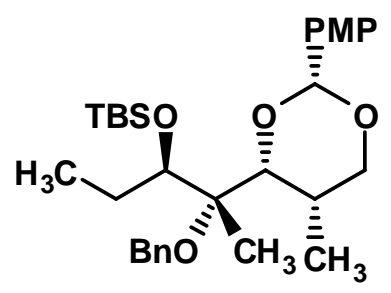

17

To a suspension of $6 \mathrm{mg}(0.261 \mathrm{mmol})$ of sodium hydride in $2 \mathrm{~mL}$ of anhydrous DMF was added $21 \mathrm{mg}(0.049 \mathrm{mmol})$ of the above PMP acetal at $0^{\circ} \mathrm{C}$. The reaction was let to warm to rt over 10 minutes and stirred at $\mathrm{rt}$ for 30 minutes. $0.05 \mathrm{ml}(0.420 \mathrm{mmol})$ of benzyl bromide (purified using basic alumina column), $17 \mathrm{mg}(0.045 \mathrm{mmol})$ of tetrabutylammonium iodide and $0.5 \mathrm{~mL}$ of HMPA were added at rt. After stirring for $4 \mathrm{~h}, 5 \mathrm{~mL}$ of water and $10 \mathrm{~mL}$ of $\mathrm{CH}_{2} \mathrm{Cl}_{2}$ were added. The $\mathrm{CH}_{2} \mathrm{Cl}_{2}$ layer was separated and washed again using $5 \mathrm{~mL}$ of water. The aqueous layers were combined and extracted using $\mathrm{CH}_{2} \mathrm{Cl}_{2}(2 \times 5 \mathrm{~mL})$. The $\mathrm{CH}_{2} \mathrm{Cl}_{2}$ layers were combined, dried using $\mathrm{Na}_{2} \mathrm{SO}_{4}$ and evaporated. FCC using 5\% ethyl acetate-hexane gave $19 \mathrm{mg}$ of $17(76 \%)$ as a clear colorless oil $\left(\mathrm{R}_{\mathrm{f}}=0.50\right.$ in $10 \%$ ethyl acetate-hexanes). $[\alpha]_{\mathrm{D}}+7.1(c=$ $\left.0.007, \mathrm{CDCl}_{3}\right) .{ }^{1} \mathrm{H}$ and ${ }^{13} \mathrm{C}$ NMR matched identically to the known Woerpel intermediate. ${ }^{11}$

\footnotetext{
${ }^{11}$ Peng, Z.; Woerpel, K.A.; J. Am. Chem. Soc. 2003, 125, 6108
} 


\section{Stereochemical Determination of $\alpha$-hydroxy ketones}

The absolute stereochemistry of the camphor derivative of the $\alpha$-hydroxy ketone from Table 2 , entry 3 was determined by X-ray crystallography.

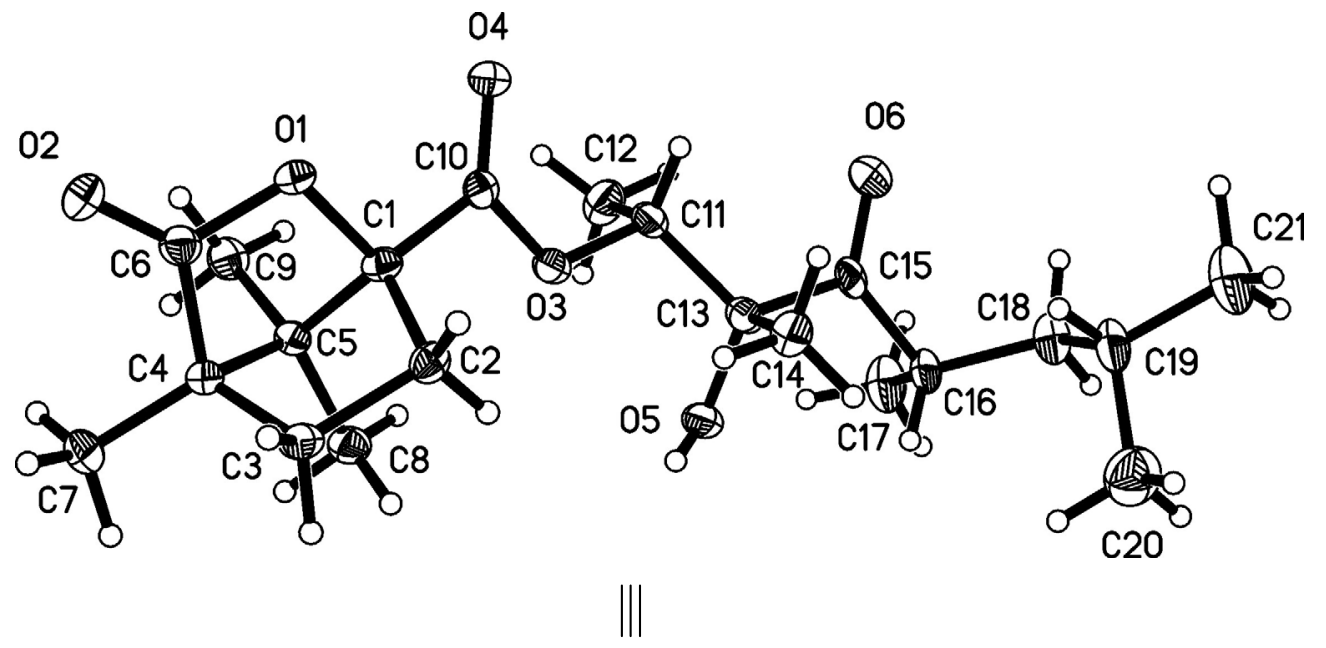<smiles>CC(C)C[C@H](C)C(=O)[C@](C)(O)[C@@H](C)OC(=O)C12CCC(C)(C(=O)C1)C2(C)C</smiles>

Table 1. Crystal data and structure refinement for $\mathrm{C}_{21} \mathrm{H}_{34} \mathrm{O}_{6}$.

Empirical formula

Formula weight

Temperature

Wavelength

Crystal system

Space group

Unit cell dimensions

Volume

$\mathrm{Z}$

Density (calculated)
C21 H34 O6

382.48

100(2) K

$0.71073 \AA$

Monoclinic

$\mathrm{P} 2(1)$

$\mathrm{a}=13.4736(8) \AA \quad \alpha=90^{\circ}$

$\mathrm{b}=5.9407(3) \AA \quad \beta=99.044(1)^{\circ}$

$\mathrm{c}=13.7189(8) \AA \quad \gamma=90^{\circ}$

1084.44(11) $\AA^{3}$

2

$1.171 \mathrm{Mg} / \mathrm{m}^{3}$ 
Absorption coefficient

$\mathrm{F}(000)$

Crystal size

Theta range for data collection

Index ranges

Reflections collected

Independent reflections

Completeness to theta $=30.60^{\circ}$

Absorption correction

Max. and min. transmission

Refinement method

Data / restraints / parameters

Goodness-of-fit on $\mathrm{F}^{2}$

Final $\mathrm{R}$ indices [I>2sigma(I)]

$\mathrm{R}$ indices (all data)

Absolute structure parameter

Largest diff. peak and hole
$0.084 \mathrm{~mm}^{-1}$

416

$0.35 \times 0.13 \times 0.05 \mathrm{~mm}^{3}$

1.97 to $30.60^{\circ}$.

$-19<=\mathrm{h}<=19,-8<=\mathrm{k}<=8,-19<=\mathrm{l}<=19$

13386

$6527[\mathrm{R}(\mathrm{int})=0.0303]$

$99.7 \%$

Semi-empirical from equivalents

0.9958 and 0.9711

Full-matrix least-squares on $\mathrm{F}^{2}$

6527 / 1 / 380

1.007

$\mathrm{R} 1=0.0505, \mathrm{wR} 2=0.1135$

$\mathrm{R} 1=0.0579, \mathrm{wR} 2=0.1175$

$-0.54(73)$

0.396 and -0.206 e. $\AA^{-3}$ 
Table 2. Atomic coordinates $\left(x \mathbf{1 0}^{4}\right)$ and equivalent isotropic displacement parameters $\left(\AA^{2} \times 10^{3}\right)$

$\mathrm{U}(\mathrm{eq})$ is defined as one third of the trace of the orthogonalized $\mathrm{U}^{\mathrm{ij}}$ tensor.

\begin{tabular}{|c|c|c|c|c|}
\hline & $\mathrm{x}$ & $\mathrm{y}$ & $\mathrm{z}$ & $\mathrm{U}(\mathrm{eq})$ \\
\hline $\mathrm{O}(1)$ & $1920(1)$ & $-7241(2)$ & $-375(1)$ & $15(1)$ \\
\hline $\mathrm{O}(2)$ & $3446(1)$ & $-7663(2)$ & $548(1)$ & $22(1)$ \\
\hline $\mathrm{O}(3)$ & $170(1)$ & $-4679(2)$ & $-2273(1)$ & $19(1)$ \\
\hline $\mathrm{O}(4)$ & $310(1)$ & $-8294(2)$ & $-1753(1)$ & $22(1)$ \\
\hline $\mathrm{O}(5)$ & $-770(1)$ & $-1076(2)$ & $-3323(1)$ & $20(1)$ \\
\hline $\mathrm{O}(6)$ & $-2684(1)$ & $-4960(2)$ & $-3944(1)$ & $29(1)$ \\
\hline $\mathrm{C}(1)$ & 1458(1) & $-5421(2)$ & $-997(1)$ & 14(1) \\
\hline$C(2)$ & $1215(1)$ & $-3572(3)$ & $-288(1)$ & $16(1)$ \\
\hline $\mathrm{C}(3)$ & 2284(1) & $-2760(3)$ & $157(1)$ & $18(1)$ \\
\hline $\mathrm{C}(4)$ & 2993(1) & $-4175(3)$ & $-397(1)$ & $16(1)$ \\
\hline$C(5)$ & $2362(1)$ & $-4452(3)$ & $-1445(1)$ & $16(1)$ \\
\hline$C(6)$ & $2879(1)$ & $-6523(3)$ & $-3(1)$ & $16(1)$ \\
\hline $\mathrm{C}(7)$ & 4064(1) & $-3346(3)$ & $-314(2)$ & $24(1)$ \\
\hline $\mathrm{C}(8)$ & $2158(1)$ & $-2253(3)$ & $-2015(1)$ & $20(1)$ \\
\hline $\mathrm{C}(9)$ & 2814(1) & $-6169(3)$ & $-2084(1)$ & 21(1) \\
\hline$C(10)$ & $583(1)$ & $-6353(3)$ & $-1710(1)$ & $14(1)$ \\
\hline $\mathrm{C}(11)$ & $-713(1)$ & $-5113(3)$ & $-3022(1)$ & $17(1)$ \\
\hline$C(12)$ & $-359(1)$ & $-5633(3)$ & $-3989(1)$ & $25(1)$ \\
\hline$C(13)$ & $-1323(1)$ & $-2923(3)$ & $-3022(1)$ & $16(1)$ \\
\hline$C(14)$ & $-1626(1)$ & $-2507(3)$ & $-2008(1)$ & $22(1)$ \\
\hline$C(15)$ & $-2312(1)$ & $-3126(3)$ & $-3749(1)$ & $17(1)$ \\
\hline$C(16)$ & $-2795(1)$ & $-959(3)$ & $-4181(1)$ & $20(1)$ \\
\hline $\mathrm{C}(17)$ & $-2506(2)$ & $-698(4)$ & $-5219(1)$ & $31(1)$ \\
\hline $\mathrm{C}(18)$ & $-3938(1)$ & $-1042(4)$ & $-4228(1)$ & $25(1)$ \\
\hline C(19) & $-4288(1)$ & $-821(4)$ & $-3223(1)$ & $26(1)$ \\
\hline$C(20)$ & $-4158(2)$ & $1569(4)$ & $-2822(2)$ & $39(1)$ \\
\hline $\mathrm{C}(21)$ & $-5384(2)$ & $-1563(5)$ & $-3302(2)$ & $43(1)$ \\
\hline
\end{tabular}


Table 3. Bond lengths $[\AA ̊ \AA]$ and angles $\left[{ }^{\circ}\right]$

\begin{tabular}{|c|c|c|c|}
\hline $\mathrm{O}(1)-\mathrm{C}(6)$ & $1.3796(19)$ & $\mathrm{C}(9)-\mathrm{H}(9 \mathrm{C})$ & $0.91(2)$ \\
\hline $\mathrm{O}(1)-\mathrm{C}(1)$ & $1.4554(18)$ & $\mathrm{C}(11)-\mathrm{C}(12)$ & $1.510(2)$ \\
\hline $\mathrm{O}(2)-\mathrm{C}(6)$ & 1.1971(19) & $\mathrm{C}(11)-\mathrm{C}(13)$ & $1.539(2)$ \\
\hline $\mathrm{O}(3)-\mathrm{C}(10)$ & $1.3263(19)$ & $\mathrm{C}(11)-\mathrm{H}(11)$ & $0.940(19)$ \\
\hline $\mathrm{O}(3)-\mathrm{C}(11)$ & $1.4678(19)$ & $\mathrm{C}(12)-\mathrm{H}(12 \mathrm{~A})$ & $0.95(3)$ \\
\hline $\mathrm{O}(4)-\mathrm{C}(10)$ & $1.2091(19)$ & $\mathrm{C}(12)-\mathrm{H}(12 \mathrm{~B})$ & $0.91(3)$ \\
\hline $\mathrm{O}(5)-\mathrm{C}(13)$ & $1.4231(19)$ & $\mathrm{C}(12)-\mathrm{H}(12 \mathrm{C})$ & $0.95(2)$ \\
\hline $\mathrm{O}(5)-\mathrm{H}(5 \mathrm{O})$ & $0.77(3)$ & $\mathrm{C}(13)-\mathrm{C}(14)$ & $1.530(2)$ \\
\hline $\mathrm{O}(6)-\mathrm{C}(15)$ & $1.212(2)$ & $\mathrm{C}(13)-\mathrm{C}(15)$ & $1.538(2)$ \\
\hline C(1)-C(10) & $1.513(2)$ & $\mathrm{C}(14)-\mathrm{H}(14 \mathrm{~A})$ & $0.98(2)$ \\
\hline $\mathrm{C}(1)-\mathrm{C}(2)$ & $1.536(2)$ & $\mathrm{C}(14)-\mathrm{H}(14 \mathrm{~B})$ & $0.92(2)$ \\
\hline $\mathrm{C}(1)-\mathrm{C}(5)$ & $1.559(2)$ & $\mathrm{C}(14)-\mathrm{H}(14 \mathrm{C})$ & $0.98(2)$ \\
\hline$C(2)-C(3)$ & $1.551(2)$ & $C(15)-C(16)$ & $1.520(2)$ \\
\hline $\mathrm{C}(2)-\mathrm{H}(2 \mathrm{~A})$ & $0.93(2)$ & $\mathrm{C}(16)-\mathrm{C}(18)$ & $1.533(2)$ \\
\hline $\mathrm{C}(2)-\mathrm{H}(2 \mathrm{~B})$ & $0.96(2)$ & $\mathrm{C}(16)-\mathrm{C}(17)$ & $1.541(3)$ \\
\hline$C(3)-C(4)$ & $1.557(2)$ & $\mathrm{C}(16)-\mathrm{H}(16)$ & $0.95(2)$ \\
\hline $\mathrm{C}(3)-\mathrm{H}(3 \mathrm{~A})$ & $0.96(2)$ & $\mathrm{C}(17)-\mathrm{H}(17 \mathrm{~A})$ & $0.99(3)$ \\
\hline $\mathrm{C}(3)-\mathrm{H}(3 \mathrm{~B})$ & $0.950(19)$ & $\mathrm{C}(17)-\mathrm{H}(17 \mathrm{~B})$ & $0.98(3)$ \\
\hline $\mathrm{C}(4)-\mathrm{C}(7)$ & $1.512(2)$ & $\mathrm{C}(17)-\mathrm{H}(17 \mathrm{C})$ & $0.97(3)$ \\
\hline$C(4)-C(6)$ & $1.513(2)$ & $\mathrm{C}(18)-\mathrm{C}(19)$ & $1.531(3)$ \\
\hline$C(4)-C(5)$ & $1.559(2)$ & $\mathrm{C}(18)-\mathrm{H}(18 \mathrm{~A})$ & $0.99(3)$ \\
\hline$C(5)-C(8)$ & $1.525(2)$ & $\mathrm{C}(18)-\mathrm{H}(18 \mathrm{~B})$ & $1.00(2)$ \\
\hline$C(5)-C(9)$ & $1.532(2)$ & $\mathrm{C}(19)-\mathrm{C}(20)$ & $1.522(3)$ \\
\hline $\mathrm{C}(7)-\mathrm{H}(7 \mathrm{~A})$ & $0.97(2)$ & $\mathrm{C}(19)-\mathrm{C}(21)$ & $1.529(3)$ \\
\hline $\mathrm{C}(7)-\mathrm{H}(7 \mathrm{~B})$ & $0.93(2)$ & $\mathrm{C}(19)-\mathrm{H}(19)$ & $0.98(2)$ \\
\hline $\mathrm{C}(7)-\mathrm{H}(7 \mathrm{C})$ & $0.96(3)$ & $\mathrm{C}(20)-\mathrm{H}(20 \mathrm{~A})$ & $0.95(3)$ \\
\hline $\mathrm{C}(8)-\mathrm{H}(8 \mathrm{~A})$ & $0.93(2)$ & $\mathrm{C}(20)-\mathrm{H}(20 \mathrm{~B})$ & $1.00(3)$ \\
\hline $\mathrm{C}(8)-\mathrm{H}(8 \mathrm{~B})$ & $0.93(3)$ & $\mathrm{C}(20)-\mathrm{H}(20 \mathrm{C})$ & $0.97(3)$ \\
\hline $\mathrm{C}(8)-\mathrm{H}(8 \mathrm{C})$ & $0.97(2)$ & $\mathrm{C}(21)-\mathrm{H}(21 \mathrm{~A})$ & $1.01(4)$ \\
\hline $\mathrm{C}(9)-\mathrm{H}(9 \mathrm{~A})$ & $0.94(2)$ & $\mathrm{C}(21)-\mathrm{H}(21 \mathrm{~B})$ & $0.94(4)$ \\
\hline $\mathrm{C}(9)-\mathrm{H}(9 \mathrm{~B})$ & $0.94(2)$ & $\mathrm{C}(21)-\mathrm{H}(21 \mathrm{C})$ & $0.94(3)$ \\
\hline $\mathrm{C}(6)-\mathrm{O}(1)-\mathrm{C}(1)$ & $106.05(11)$ & $\mathrm{C}(13)-\mathrm{O}(5)-\mathrm{H}(5 \mathrm{O})$ & 104.1(19) \\
\hline $\mathrm{C}(10)-\mathrm{O}(3)-\mathrm{C}(11)$ & 119.70(12) & $\mathrm{O}(1)-\mathrm{C}(1)-\mathrm{C}(10)$ & 109.00(12) \\
\hline
\end{tabular}




\begin{tabular}{|c|c|c|c|}
\hline $\mathrm{O}(1)-\mathrm{C}(1)-\mathrm{C}(2)$ & $105.89(12)$ & $\mathrm{H}(7 \mathrm{~A})-\mathrm{C}(7)-\mathrm{H}(7 \mathrm{C})$ & $109(2)$ \\
\hline $\mathrm{C}(10)-\mathrm{C}(1)-\mathrm{C}(2)$ & $116.72(12)$ & $\mathrm{H}(7 \mathrm{~B})-\mathrm{C}(7)-\mathrm{H}(7 \mathrm{C})$ & $111(2)$ \\
\hline $\mathrm{O}(1)-\mathrm{C}(1)-\mathrm{C}(5)$ & $102.52(11)$ & $\mathrm{C}(5)-\mathrm{C}(8)-\mathrm{H}(8 \mathrm{~A})$ & $111.5(14)$ \\
\hline $\mathrm{C}(10)-\mathrm{C}(1)-\mathrm{C}(5)$ & $117.23(12)$ & $\mathrm{C}(5)-\mathrm{C}(8)-\mathrm{H}(8 \mathrm{~B})$ & $110.5(15)$ \\
\hline$C(2)-C(1)-C(5)$ & $104.04(12)$ & $\mathrm{H}(8 \mathrm{~A})-\mathrm{C}(8)-\mathrm{H}(8 \mathrm{~B})$ & $104.9(19)$ \\
\hline $\mathrm{C}(1)-\mathrm{C}(2)-\mathrm{C}(3)$ & 101.23(12) & $\mathrm{C}(5)-\mathrm{C}(8)-\mathrm{H}(8 \mathrm{C})$ & $115.2(14)$ \\
\hline $\mathrm{C}(1)-\mathrm{C}(2)-\mathrm{H}(2 \mathrm{~A})$ & $110.0(13)$ & $\mathrm{H}(8 \mathrm{~A})-\mathrm{C}(8)-\mathrm{H}(8 \mathrm{C})$ & $110.6(18)$ \\
\hline $\mathrm{C}(3)-\mathrm{C}(2)-\mathrm{H}(2 \mathrm{~A})$ & $112.5(12)$ & $\mathrm{H}(8 \mathrm{~B})-\mathrm{C}(8)-\mathrm{H}(8 \mathrm{C})$ & $103(2)$ \\
\hline $\mathrm{C}(1)-\mathrm{C}(2)-\mathrm{H}(2 \mathrm{~B})$ & $108.2(13)$ & $\mathrm{C}(5)-\mathrm{C}(9)-\mathrm{H}(9 \mathrm{~A})$ & $110.2(15)$ \\
\hline $\mathrm{C}(3)-\mathrm{C}(2)-\mathrm{H}(2 \mathrm{~B})$ & $113.6(14)$ & $\mathrm{C}(5)-\mathrm{C}(9)-\mathrm{H}(9 \mathrm{~B})$ & $110.1(13)$ \\
\hline $\mathrm{H}(2 \mathrm{~A})-\mathrm{C}(2)-\mathrm{H}(2 \mathrm{~B})$ & $110.7(18)$ & $\mathrm{H}(9 \mathrm{~A})-\mathrm{C}(9)-\mathrm{H}(9 \mathrm{~B})$ & $104.2(18)$ \\
\hline $\mathrm{C}(2)-\mathrm{C}(3)-\mathrm{C}(4)$ & $104.06(12)$ & $\mathrm{C}(5)-\mathrm{C}(9)-\mathrm{H}(9 \mathrm{C})$ & $109.9(14)$ \\
\hline $\mathrm{C}(2)-\mathrm{C}(3)-\mathrm{H}(3 \mathrm{~A})$ & $111.7(13)$ & $\mathrm{H}(9 \mathrm{~A})-\mathrm{C}(9)-\mathrm{H}(9 \mathrm{C})$ & $106(2)$ \\
\hline $\mathrm{C}(4)-\mathrm{C}(3)-\mathrm{H}(3 \mathrm{~A})$ & $107.2(13)$ & H(9B)-C(9)-H(9C) & $116(2)$ \\
\hline $\mathrm{C}(2)-\mathrm{C}(3)-\mathrm{H}(3 \mathrm{~B})$ & $114.4(12)$ & $\mathrm{O}(4)-\mathrm{C}(10)-\mathrm{O}(3)$ & $126.18(14)$ \\
\hline $\mathrm{C}(4)-\mathrm{C}(3)-\mathrm{H}(3 \mathrm{~B})$ & $111.7(12)$ & $\mathrm{O}(4)-\mathrm{C}(10)-\mathrm{C}(1)$ & $125.49(14)$ \\
\hline $\mathrm{H}(3 \mathrm{~A})-\mathrm{C}(3)-\mathrm{H}(3 \mathrm{~B})$ & $107.6(19)$ & $\mathrm{O}(3)-\mathrm{C}(10)-\mathrm{C}(1)$ & $108.33(12)$ \\
\hline$C(7)-C(4)-C(6)$ & $115.01(14)$ & $\mathrm{O}(3)-\mathrm{C}(11)-\mathrm{C}(12)$ & $108.55(13)$ \\
\hline $\mathrm{C}(7)-\mathrm{C}(4)-\mathrm{C}(3)$ & $116.01(14)$ & $\mathrm{O}(3)-\mathrm{C}(11)-\mathrm{C}(13)$ & $103.03(12)$ \\
\hline$C(6)-C(4)-C(3)$ & 102.63(13) & $C(12)-C(11)-C(13)$ & $114.58(14)$ \\
\hline$C(7)-C(4)-C(5)$ & 118.68(14) & $\mathrm{O}(3)-\mathrm{C}(11)-\mathrm{H}(11)$ & $108.3(11)$ \\
\hline$C(6)-C(4)-C(5)$ & $99.23(12)$ & $\mathrm{C}(12)-\mathrm{C}(11)-\mathrm{H}(11)$ & $111.6(11)$ \\
\hline$C(3)-C(4)-C(5)$ & $102.69(12)$ & $\mathrm{C}(13)-\mathrm{C}(11)-\mathrm{H}(11)$ & $110.3(11)$ \\
\hline $\mathrm{C}(8)-\mathrm{C}(5)-\mathrm{C}(9)$ & $109.45(13)$ & $\mathrm{C}(11)-\mathrm{C}(12)-\mathrm{H}(12 \mathrm{~A})$ & $108.6(17)$ \\
\hline $\mathrm{C}(8)-\mathrm{C}(5)-\mathrm{C}(1)$ & $115.42(13)$ & $\mathrm{C}(11)-\mathrm{C}(12)-\mathrm{H}(12 \mathrm{~B})$ & 109.3(15) \\
\hline $\mathrm{C}(9)-\mathrm{C}(5)-\mathrm{C}(1)$ & $112.65(13)$ & $\mathrm{H}(12 \mathrm{~A})-\mathrm{C}(12)-\mathrm{H}(12 \mathrm{~B})$ & $109(2)$ \\
\hline $\mathrm{C}(8)-\mathrm{C}(5)-\mathrm{C}(4)$ & $114.23(13)$ & $\mathrm{C}(11)-\mathrm{C}(12)-\mathrm{H}(12 \mathrm{C})$ & $110.8(14)$ \\
\hline $\mathrm{C}(9)-\mathrm{C}(5)-\mathrm{C}(4)$ & 112.91(13) & $\mathrm{H}(12 \mathrm{~A})-\mathrm{C}(12)-\mathrm{H}(12 \mathrm{C})$ & $110(2)$ \\
\hline $\mathrm{C}(1)-\mathrm{C}(5)-\mathrm{C}(4)$ & $91.29(11)$ & $\mathrm{H}(12 \mathrm{~B})-\mathrm{C}(12)-\mathrm{H}(12 \mathrm{C})$ & $109(2)$ \\
\hline $\mathrm{O}(2)-\mathrm{C}(6)-\mathrm{O}(1)$ & $121.74(14)$ & $\mathrm{O}(5)-\mathrm{C}(13)-\mathrm{C}(14)$ & $111.45(14)$ \\
\hline $\mathrm{O}(2)-\mathrm{C}(6)-\mathrm{C}(4)$ & $131.05(15)$ & $\mathrm{O}(5)-\mathrm{C}(13)-\mathrm{C}(15)$ & $108.15(12)$ \\
\hline $\mathrm{O}(1)-\mathrm{C}(6)-\mathrm{C}(4)$ & 107.17(12) & $\mathrm{C}(14)-\mathrm{C}(13)-\mathrm{C}(15)$ & $105.88(12)$ \\
\hline $\mathrm{C}(4)-\mathrm{C}(7)-\mathrm{H}(7 \mathrm{~A})$ & $111.0(12)$ & $\mathrm{O}(5)-\mathrm{C}(13)-\mathrm{C}(11)$ & $110.36(12)$ \\
\hline $\mathrm{C}(4)-\mathrm{C}(7)-\mathrm{H}(7 \mathrm{~B})$ & $109.0(14)$ & $\mathrm{C}(14)-\mathrm{C}(13)-\mathrm{C}(11)$ & $110.85(13)$ \\
\hline $\mathrm{H}(7 \mathrm{~A})-\mathrm{C}(7)-\mathrm{H}(7 \mathrm{~B})$ & $109.7(18)$ & $\mathrm{C}(15)-\mathrm{C}(13)-\mathrm{C}(11)$ & $110.01(13)$ \\
\hline $\mathrm{C}(4)-\mathrm{C}(7)-\mathrm{H}(7 \mathrm{C})$ & $107.7(18)$ & $\mathrm{C}(13)-\mathrm{C}(14)-\mathrm{H}(14 \mathrm{~A})$ & 109.3(13) \\
\hline
\end{tabular}




$\begin{array}{llll}\mathrm{C}(13)-\mathrm{C}(14)-\mathrm{H}(14 \mathrm{~B}) & 109.6(15) & \mathrm{C}(16)-\mathrm{C}(18)-\mathrm{H}(18 \mathrm{~A}) & 108.6(14) \\ \mathrm{H}(14 \mathrm{~A})-\mathrm{C}(14)-\mathrm{H}(14 \mathrm{~B}) & 112(2) & \mathrm{C}(19)-\mathrm{C}(18)-\mathrm{H}(18 \mathrm{~B}) & 110.9(13) \\ \mathrm{C}(13)-\mathrm{C}(14)-\mathrm{H}(14 \mathrm{C}) & 111.5(13) & \mathrm{C}(16)-\mathrm{C}(18)-\mathrm{H}(18 \mathrm{~B}) & 106.4(13) \\ \mathrm{H}(14 \mathrm{~A})-\mathrm{C}(14)-\mathrm{H}(14 \mathrm{C}) & 110.9(18) & \mathrm{H}(18 \mathrm{~A})-\mathrm{C}(18)-\mathrm{H}(18 \mathrm{~B}) & 107.4(18) \\ \mathrm{H}(14 \mathrm{~B})-\mathrm{C}(14)-\mathrm{H}(14 \mathrm{C}) & 103(2) & \mathrm{C}(20)-\mathrm{C}(19)-\mathrm{C}(21) & 110.45(19) \\ \mathrm{O}(6)-\mathrm{C}(15)-\mathrm{C}(16) & 122.57(14) & \mathrm{C}(20)-\mathrm{C}(19)-\mathrm{C}(18) & 111.78(17) \\ \mathrm{O}(6)-\mathrm{C}(15)-\mathrm{C}(13) & 119.97(15) & \mathrm{C}(21)-\mathrm{C}(19)-\mathrm{C}(18) & 110.02(17) \\ \mathrm{C}(16)-\mathrm{C}(15)-\mathrm{C}(13) & 117.45(14) & \mathrm{C}(20)-\mathrm{C}(19)-\mathrm{H}(19) & 104.1(13) \\ \mathrm{C}(15)-\mathrm{C}(16)-\mathrm{C}(18) & 110.79(15) & \mathrm{C}(21)-\mathrm{C}(19)-\mathrm{H}(19) & 109.3(14) \\ \mathrm{C}(15)-\mathrm{C}(16)-\mathrm{C}(17) & 106.94(15) & \mathrm{C}(18)-\mathrm{C}(19)-\mathrm{H}(19) & 111.1(13) \\ \mathrm{C}(18)-\mathrm{C}(16)-\mathrm{C}(17) & 111.29(14) & \mathrm{C}(19)-\mathrm{C}(20)-\mathrm{H}(20 \mathrm{~A}) & 109(2) \\ \mathrm{C}(15)-\mathrm{C}(16)-\mathrm{H}(16) & 107.2(14) & \mathrm{C}(19)-\mathrm{C}(20)-\mathrm{H}(20 \mathrm{~B}) & 108.4(19) \\ \mathrm{C}(18)-\mathrm{C}(16)-\mathrm{H}(16) & 113.3(14) & \mathrm{H}(20 \mathrm{~A})-\mathrm{C}(20)-\mathrm{H}(20 \mathrm{~B}) & 107(3) \\ \mathrm{C}(17)-\mathrm{C}(16)-\mathrm{H}(16) & 107.0(14) & \mathrm{C}(19)-\mathrm{C}(20)-\mathrm{H}(20 \mathrm{C}) & 111.6(17) \\ \mathrm{C}(16)-\mathrm{C}(17)-\mathrm{H}(17 \mathrm{~A}) & 108.9(14) & \mathrm{H}(20 \mathrm{~A})-\mathrm{C}(20)-\mathrm{H}(20 \mathrm{C}) & 109(2) \\ \mathrm{C}(16)-\mathrm{C}(17)-\mathrm{H}(17 \mathrm{~B}) & 114.0(15) & \mathrm{H}(20 \mathrm{~B})-\mathrm{C}(20)-\mathrm{H}(20 \mathrm{C}) & 112(2) \\ \mathrm{H}(17 \mathrm{~A})-\mathrm{C}(17)-\mathrm{H}(17 \mathrm{~B}) & 111(2) & \mathrm{C}(19)-\mathrm{C}(21)-\mathrm{H}(21 \mathrm{~A}) & 112(2) \\ \mathrm{C}(16)-\mathrm{C}(17)-\mathrm{H}(17 \mathrm{C}) & 112.9(16) & \mathrm{C}(19)-\mathrm{C}(21)-\mathrm{H}(21 \mathrm{~B}) & 113(2) \\ \mathrm{H}(17 \mathrm{~A})-\mathrm{C}(17)-\mathrm{H}(17 \mathrm{C}) & 105(2) & \mathrm{H}(21 \mathrm{~A})-\mathrm{C}(21)-\mathrm{H}(21 \mathrm{~B}) & 106(3) \\ \mathrm{H}(17 \mathrm{~B})-\mathrm{C}(17)-\mathrm{H}(17 \mathrm{C}) & 105(2) & \mathrm{C}(19)-\mathrm{C}(21)-\mathrm{H}(21 \mathrm{C}) & 111.0(19) \\ \mathrm{C}(19)-\mathrm{C}(18)-\mathrm{C}(16) & 114.19(14) & \mathrm{H}(21 \mathrm{~A})-\mathrm{C}(21)-\mathrm{H}(21 \mathrm{C}) & 100(3) \\ \mathrm{C}(19)-\mathrm{C}(18)-\mathrm{H}(18 \mathrm{~A}) & 109.0(13) & & 115(3) \\ & & & \end{array}$


Table 4. Anisotropic displacement parameters $\left(\AA^{2} \times 10^{3}\right)$. The anisotropic displacement factor exponent takes the form: $-2 \pi^{2}\left[h^{2} a^{* 2} U^{11}+\ldots+2 h k a^{*} b^{*} U^{12}\right]$

\begin{tabular}{lcccccc}
\hline & $\mathrm{U} 11$ & $\mathrm{U}^{22}$ & $\mathrm{U}^{33}$ & $\mathrm{U}^{23}$ & $\mathrm{U} 13$ & $\mathrm{U} 12$ \\
\hline $\mathrm{O}(1)$ & $16(1)$ & $10(1)$ & $20(1)$ & $3(1)$ & $2(1)$ & $-1(1)$ \\
$\mathrm{O}(2)$ & $21(1)$ & $16(1)$ & $28(1)$ & $3(1)$ & $-1(1)$ & $3(1)$ \\
$\mathrm{O}(3)$ & $15(1)$ & $13(1)$ & $26(1)$ & $4(1)$ & $-3(1)$ & $-2(1)$ \\
$\mathrm{O}(4)$ & $21(1)$ & $13(1)$ & $29(1)$ & $1(1)$ & $-1(1)$ & $-3(1)$ \\
$\mathrm{O}(5)$ & $17(1)$ & $16(1)$ & $26(1)$ & $1(1)$ & $-1(1)$ & $-4(1)$ \\
$\mathrm{O}(6)$ & $21(1)$ & $23(1)$ & $40(1)$ & $-5(1)$ & $-1(1)$ & $-5(1)$ \\
$\mathrm{C}(1)$ & $15(1)$ & $9(1)$ & $17(1)$ & $1(1)$ & $2(1)$ & $0(1)$ \\
$\mathrm{C}(2)$ & $16(1)$ & $12(1)$ & $21(1)$ & $-1(1)$ & $4(1)$ & $1(1)$ \\
$\mathrm{C}(3)$ & $21(1)$ & $11(1)$ & $22(1)$ & $-3(1)$ & $1(1)$ & $0(1)$ \\
$\mathrm{C}(4)$ & $14(1)$ & $11(1)$ & $24(1)$ & $1(1)$ & $2(1)$ & $0(1)$ \\
$\mathrm{C}(5)$ & $15(1)$ & $12(1)$ & $20(1)$ & $2(1)$ & $4(1)$ & $-1(1)$ \\
$\mathrm{C}(6)$ & $17(1)$ & $13(1)$ & $20(1)$ & $-1(1)$ & $3(1)$ & $1(1)$ \\
$\mathrm{C}(7)$ & $17(1)$ & $19(1)$ & $34(1)$ & $3(1)$ & $0(1)$ & $-4(1)$ \\
$\mathrm{C}(8)$ & $19(1)$ & $15(1)$ & $24(1)$ & $5(1)$ & $4(1)$ & $-2(1)$ \\
$\mathrm{C}(9)$ & $20(1)$ & $19(1)$ & $25(1)$ & $0(1)$ & $8(1)$ & $-1(1)$ \\
$\mathrm{C}(10)$ & $13(1)$ & $14(1)$ & $17(1)$ & $0(1)$ & $3(1)$ & $0(1)$ \\
$\mathrm{C}(11)$ & $15(1)$ & $15(1)$ & $19(1)$ & $0(1)$ & $0(1)$ & $-2(1)$ \\
$\mathrm{C}(12)$ & $24(1)$ & $26(1)$ & $25(1)$ & $-3(1)$ & $6(1)$ & $4(1)$ \\
$\mathrm{C}(13)$ & $13(1)$ & $15(1)$ & $18(1)$ & $-1(1)$ & $1(1)$ & $-2(1)$ \\
$\mathrm{C}(14)$ & $23(1)$ & $26(1)$ & $18(1)$ & $-2(1)$ & $3(1)$ & $3(1)$ \\
$\mathrm{C}(15)$ & $13(1)$ & $21(1)$ & $18(1)$ & $-1(1)$ & $3(1)$ & $-2(1)$ \\
$\mathrm{C}(16)$ & $14(1)$ & $25(1)$ & $20(1)$ & $4(1)$ & $3(1)$ & $1(1)$ \\
$\mathrm{C}(17)$ & $23(1)$ & $48(1)$ & $24(1)$ & $11(1)$ & $6(1)$ & $4(1)$ \\
$\mathrm{C}(18)$ & $14(1)$ & $36(1)$ & $24(1)$ & $1(1)$ & $2(1)$ & $2(1)$ \\
$\mathrm{C}(19)$ & $17(1)$ & $35(1)$ & $28(1)$ & $5(1)$ & $5(1)$ & $0(1)$ \\
$\mathrm{C}(20)$ & $40(1)$ & $43(1)$ & $38(1)$ & $-7(1)$ & $13(1)$ & $1(1)$ \\
$\mathrm{C}(21)$ & $21(1)$ & $60(2)$ & $52(1)$ & $3(1)$ & $13(1)$ & $-5(1)$ \\
& & & & & & \\
\hline
\end{tabular}


Table 5. Hydrogen coordinates ( $\left.\times 10^{4}\right)$ and isotropic displacement parameters $\left(\AA^{2} \times 103\right)$

\begin{tabular}{|c|c|c|c|c|}
\hline & $\mathrm{x}$ & $\mathrm{y}$ & $\mathrm{z}$ & $\mathrm{U}(\mathrm{eq})$ \\
\hline $\mathrm{H}(5 \mathrm{O})$ & $-462(19)$ & $-650(40)$ & $-2841(19)$ & $28(6)$ \\
\hline $\mathrm{H}(2 \mathrm{~A})$ & $871(15)$ & $-4180(40)$ & $183(15)$ & $16(5)$ \\
\hline $\mathrm{H}(2 \mathrm{~B})$ & $817(17)$ & $-2440(40)$ & $-668(16)$ & $23(5)$ \\
\hline $\mathrm{H}(3 \mathrm{~A})$ & $2386(17)$ & $-1210(40)$ & $8(16)$ & $24(6)$ \\
\hline $\mathrm{H}(3 \mathrm{~B})$ & $2446(14)$ & $-2940(40)$ & $853(14)$ & $13(4)$ \\
\hline $\mathrm{H}(7 \mathrm{~A})$ & $4440(15)$ & $-4240(40)$ & $-720(14)$ & $14(5)$ \\
\hline H(7B) & $4368(16)$ & $-3430(40)$ & $344(16)$ & $23(5)$ \\
\hline $\mathrm{H}(7 \mathrm{C})$ & $4040(20)$ & $-1820(60)$ & $-540(20)$ & $52(8)$ \\
\hline $\mathrm{H}(8 \mathrm{~A})$ & $1774(15)$ & $-2490(40)$ & $-2635(15)$ & $16(5)$ \\
\hline $\mathrm{H}(8 \mathrm{~B})$ & 2757(19) & $-1620(40)$ & $-2145(18)$ & $31(6)$ \\
\hline $\mathrm{H}(8 \mathrm{C})$ & $1865(17)$ & $-1060(40)$ & $-1664(16)$ & $26(6)$ \\
\hline $\mathrm{H}(9 \mathrm{~A})$ & $3386(18)$ & $-5560(40)$ & $-2302(16)$ & $25(6)$ \\
\hline H(9B) & $2363(17)$ & $-6450(40)$ & $-2670(16)$ & $20(5)$ \\
\hline $\mathrm{H}(9 \mathrm{C})$ & $3036(17)$ & $-7400(40)$ & $-1714(16)$ & $25(5)$ \\
\hline $\mathrm{H}(11)$ & $-1071(14)$ & $-6320(30)$ & $-2807(13)$ & $8(4)$ \\
\hline $\mathrm{H}(12 \mathrm{~A})$ & $80(20)$ & $-6910(50)$ & $-3900(20)$ & $50(8)$ \\
\hline $\mathrm{H}(12 \mathrm{~B})$ & $-14(18)$ & $-4430(50)$ & $-4174(17)$ & $31(6)$ \\
\hline $\mathrm{H}(12 \mathrm{C})$ & $-916(18)$ & $-5940(40)$ & $-4493(17)$ & $27(6)$ \\
\hline $\mathrm{H}(14 \mathrm{~A})$ & $-2029(17)$ & $-1130(40)$ & $-2032(16)$ & $26(6)$ \\
\hline $\mathrm{H}(14 \mathrm{~B})$ & $-1062(19)$ & $-2430(40)$ & $-1538(18)$ & $30(6)$ \\
\hline $\mathrm{H}(14 \mathrm{C})$ & $-1996(18)$ & $-3800(40)$ & $-1794(16)$ & $26(6)$ \\
\hline $\mathrm{H}(16)$ & $-2487(17)$ & $240(40)$ & $-3792(16)$ & $27(6)$ \\
\hline $\mathrm{H}(17 \mathrm{~A})$ & $-2855(18)$ & $-1880(40)$ & $-5657(18)$ & $29(6)$ \\
\hline $\mathrm{H}(17 \mathrm{~B})$ & $-1780(20)$ & $-730(50)$ & $-5228(19)$ & $42(7)$ \\
\hline $\mathrm{H}(17 \mathrm{C})$ & $-2740(20)$ & $710(50)$ & $-5530(20)$ & $41(7)$ \\
\hline $\mathrm{H}(18 \mathrm{~A})$ & $-4189(17)$ & $-2480(40)$ & $-4535(16)$ & $29(6)$ \\
\hline $\mathrm{H}(18 \mathrm{~B})$ & $-4220(17)$ & $200(40)$ & $-4683(17)$ & $26(6)$ \\
\hline H(19) & $-3873(17)$ & $-1730(40)$ & $-2725(16)$ & $23(5)$ \\
\hline $\mathrm{H}(20 \mathrm{~A})$ & $-4550(20)$ & $2570(60)$ & $-3260(20)$ & $55(8)$ \\
\hline $\mathrm{H}(20 \mathrm{~B})$ & $-3440(20)$ & $2020(60)$ & $-2800(20)$ & $48(7)$ \\
\hline $\mathrm{H}(20 \mathrm{C})$ & $-4370(20)$ & $1700(50)$ & $-2180(20)$ & $40(7)$ \\
\hline
\end{tabular}




\begin{tabular}{lllll}
$\mathrm{H}(21 \mathrm{~A})$ & $-5470(30)$ & $-3210(80)$ & $-3470(20)$ & $70(10)$ \\
$\mathrm{H}(21 \mathrm{~B})$ & $-5810(30)$ & $-770(70)$ & $-3790(30)$ & $72(11)$ \\
$\mathrm{H}(21 \mathrm{C})$ & $-5590(20)$ & $-1570(60)$ & $-2680(20)$ & $55(8)$ \\
\hline
\end{tabular}

Table 6. Torsion angles

\begin{tabular}{|c|c|c|c|}
\hline $\mathrm{C}(6)-\mathrm{O}(1)-\mathrm{C}(1)-\mathrm{C}(10)$ & $159.39(12)$ & $\mathrm{C}(1)-\mathrm{O}(1)-\mathrm{C}(6)-\mathrm{O}(2)$ & $179.24(15)$ \\
\hline $\mathrm{C}(6)-\mathrm{O}(1)-\mathrm{C}(1)-\mathrm{C}(2)$ & $-74.27(14)$ & $\mathrm{C}(1)-\mathrm{O}(1)-\mathrm{C}(6)-\mathrm{C}(4)$ & $1.15(15)$ \\
\hline $\mathrm{C}(6)-\mathrm{O}(1)-\mathrm{C}(1)-\mathrm{C}(5)$ & $34.49(14)$ & $\mathrm{C}(7)-\mathrm{C}(4)-\mathrm{C}(6)-\mathrm{O}(2)$ & $18.5(3)$ \\
\hline $\mathrm{O}(1)-\mathrm{C}(1)-\mathrm{C}(2)-\mathrm{C}(3)$ & $68.95(14)$ & $\mathrm{C}(3)-\mathrm{C}(4)-\mathrm{C}(6)-\mathrm{O}(2)$ & $-108.44(19)$ \\
\hline $\mathrm{C}(10)-\mathrm{C}(1)-\mathrm{C}(2)-\mathrm{C}(3)$ & $-169.56(13)$ & $\mathrm{C}(5)-\mathrm{C}(4)-\mathrm{C}(6)-\mathrm{O}(2)$ & $146.23(18)$ \\
\hline $\mathrm{C}(5)-\mathrm{C}(1)-\mathrm{C}(2)-\mathrm{C}(3)$ & $-38.71(14)$ & $\mathrm{C}(7)-\mathrm{C}(4)-\mathrm{C}(6)-\mathrm{O}(1)$ & $-163.69(14)$ \\
\hline $\mathrm{C}(1)-\mathrm{C}(2)-\mathrm{C}(3)-\mathrm{C}(4)$ & $2.74(15)$ & $\mathrm{C}(3)-\mathrm{C}(4)-\mathrm{C}(6)-\mathrm{O}(1)$ & $69.41(14)$ \\
\hline $\mathrm{C}(2)-\mathrm{C}(3)-\mathrm{C}(4)-\mathrm{C}(7)$ & $164.77(14)$ & $\mathrm{C}(5)-\mathrm{C}(4)-\mathrm{C}(6)-\mathrm{O}(1)$ & $-35.92(15)$ \\
\hline$C(2)-C(3)-C(4)-C(6)$ & $-68.96(15)$ & $\mathrm{C}(11)-\mathrm{O}(3)-\mathrm{C}(10)-\mathrm{O}(4)$ & $0.9(2)$ \\
\hline$C(2)-C(3)-C(4)-C(5)$ & $33.69(15)$ & $\mathrm{C}(11)-\mathrm{O}(3)-\mathrm{C}(10)-\mathrm{C}(1)$ & $-178.84(12)$ \\
\hline $\mathrm{O}(1)-\mathrm{C}(1)-\mathrm{C}(5)-\mathrm{C}(8)$ & $-170.66(13)$ & $\mathrm{O}(1)-\mathrm{C}(1)-\mathrm{C}(10)-\mathrm{O}(4)$ & $0.3(2)$ \\
\hline $\mathrm{C}(10)-\mathrm{C}(1)-\mathrm{C}(5)-\mathrm{C}(8)$ & $70.04(18)$ & $\mathrm{C}(2)-\mathrm{C}(1)-\mathrm{C}(10)-\mathrm{O}(4)$ & $-119.51(17)$ \\
\hline $\mathrm{C}(2)-\mathrm{C}(1)-\mathrm{C}(5)-\mathrm{C}(8)$ & $-60.50(16)$ & $\mathrm{C}(5)-\mathrm{C}(1)-\mathrm{C}(10)-\mathrm{O}(4)$ & $116.13(18)$ \\
\hline $\mathrm{O}(1)-\mathrm{C}(1)-\mathrm{C}(5)-\mathrm{C}(9)$ & $62.62(15)$ & $\mathrm{O}(1)-\mathrm{C}(1)-\mathrm{C}(10)-\mathrm{O}(3)$ & $-179.95(12)$ \\
\hline $\mathrm{C}(10)-\mathrm{C}(1)-\mathrm{C}(5)-\mathrm{C}(9)$ & $-56.68(18)$ & $\mathrm{C}(2)-\mathrm{C}(1)-\mathrm{C}(10)-\mathrm{O}(3)$ & $60.21(16)$ \\
\hline $\mathrm{C}(2)-\mathrm{C}(1)-\mathrm{C}(5)-\mathrm{C}(9)$ & $172.78(13)$ & $\mathrm{C}(5)-\mathrm{C}(1)-\mathrm{C}(10)-\mathrm{O}(3)$ & $-64.16(16)$ \\
\hline $\mathrm{O}(1)-\mathrm{C}(1)-\mathrm{C}(5)-\mathrm{C}(4)$ & $-52.93(12)$ & $\mathrm{C}(10)-\mathrm{O}(3)-\mathrm{C}(11)-\mathrm{C}(12)$ & $-92.64(17)$ \\
\hline$C(10)-C(1)-C(5)-C(4)$ & $-172.24(12)$ & $\mathrm{C}(10)-\mathrm{O}(3)-\mathrm{C}(11)-\mathrm{C}(13)$ & $145.49(13)$ \\
\hline$C(2)-C(1)-C(5)-C(4)$ & $57.23(13)$ & $\mathrm{O}(3)-\mathrm{C}(11)-\mathrm{C}(13)-\mathrm{O}(5)$ & $63.06(15)$ \\
\hline$C(7)-C(4)-C(5)-C(8)$ & $-64.55(19)$ & $\mathrm{C}(12)-\mathrm{C}(11)-\mathrm{C}(13)-\mathrm{O}(5)$ & $-54.65(17)$ \\
\hline$C(6)-C(4)-C(5)-C(8)$ & $170.19(13)$ & $\mathrm{O}(3)-\mathrm{C}(11)-\mathrm{C}(13)-\mathrm{C}(14)$ & $-60.91(15)$ \\
\hline $\mathrm{C}(3)-\mathrm{C}(4)-\mathrm{C}(5)-\mathrm{C}(8)$ & $64.91(16)$ & $\mathrm{C}(12)-\mathrm{C}(11)-\mathrm{C}(13)-\mathrm{C}(14)$ & $-178.62(15)$ \\
\hline $\mathrm{C}(7)-\mathrm{C}(4)-\mathrm{C}(5)-\mathrm{C}(9)$ & $61.37(19)$ & $\mathrm{O}(3)-\mathrm{C}(11)-\mathrm{C}(13)-\mathrm{C}(15)$ & $-177.68(12)$ \\
\hline$C(6)-C(4)-C(5)-C(9)$ & $-63.89(15)$ & $\mathrm{C}(12)-\mathrm{C}(11)-\mathrm{C}(13)-\mathrm{C}(15)$ & $64.61(17)$ \\
\hline $\mathrm{C}(3)-\mathrm{C}(4)-\mathrm{C}(5)-\mathrm{C}(9)$ & $-169.17(13)$ & $\mathrm{O}(5)-\mathrm{C}(13)-\mathrm{C}(15)-\mathrm{O}(6)$ & $147.04(15)$ \\
\hline$C(7)-C(4)-C(5)-C(1)$ & $176.69(14)$ & $\mathrm{C}(14)-\mathrm{C}(13)-\mathrm{C}(15)-\mathrm{O}(6)$ & $-93.40(19)$ \\
\hline$C(6)-C(4)-C(5)-C(1)$ & $51.44(12)$ & $\mathrm{C}(11)-\mathrm{C}(13)-\mathrm{C}(15)-\mathrm{O}(6)$ & $26.4(2)$ \\
\hline $\mathrm{C}(3)-\mathrm{C}(4)-\mathrm{C}(5)-\mathrm{C}(1)$ & $-53.85(13)$ & $\mathrm{O}(5)-\mathrm{C}(13)-\mathrm{C}(15)-\mathrm{C}(16)$ & $-34.08(18)$ \\
\hline
\end{tabular}




$\begin{array}{lccc}\mathrm{C}(14)-\mathrm{C}(13)-\mathrm{C}(15)-\mathrm{C}(16) & 85.49(16) & \mathrm{C}(17)-\mathrm{C}(16)-\mathrm{C}(18)-\mathrm{C}(19) & -168.27(18) \\ \mathrm{C}(11)-\mathrm{C}(13)-\mathrm{C}(15)-\mathrm{C}(16) & -154.68(13) & \mathrm{C}(16)-\mathrm{C}(18)-\mathrm{C}(19)-\mathrm{C}(20) & 72.5(2) \\ \mathrm{O}(6)-\mathrm{C}(15)-\mathrm{C}(16)-\mathrm{C}(18) & 39.6(2) & \mathrm{C}(16)-\mathrm{C}(18)-\mathrm{C}(19)-\mathrm{C}(21) & -164.40(19) \\ \mathrm{C}(13)-\mathrm{C}(15)-\mathrm{C}(16)-\mathrm{C}(18) & -139.23(15) & & \\ \mathrm{O}(6)-\mathrm{C}(15)-\mathrm{C}(16)-\mathrm{C}(17) & -81.8(2) & & \\ \mathrm{C}(13)-\mathrm{C}(15)-\mathrm{C}(16)-\mathrm{C}(17) & 99.33(17) & & \\ \mathrm{C}(15)-\mathrm{C}(16)-\mathrm{C}(18)-\mathrm{C}(19) & 72.9(2) & & \end{array}$

Table 7. Hydrogen bonds for C21H34O6 [^ and $^{\circ}$ ].

\begin{tabular}{lcccc}
\hline $\mathrm{D}-\mathrm{H} \ldots \mathrm{A}$ & $\mathrm{d}(\mathrm{D}-\mathrm{H})$ & $\mathrm{d}(\mathrm{H} \ldots \mathrm{A})$ & $\mathrm{d}(\mathrm{D} \ldots \mathrm{A})$ & $<(\mathrm{DHA})$ \\
\hline $\mathrm{O}(5)-\mathrm{H}(5 \mathrm{O}) \ldots \mathrm{O}(4) \# 1$ & $0.77(3)$ & $2.18(3)$ & $2.916(2)$ & $159(3)$ \\
$\mathrm{C}(11)-\mathrm{H}(11) \ldots \mathrm{O}(4)$ & $0.94(2)$ & $2.46(2)$ & $2.783(2)$ & $100(1)$ \\
$\mathrm{C}(16)-\mathrm{H}(16) \ldots \mathrm{O}(5)$ & $0.95(2)$ & $2.43(2)$ & $2.797(2)$ & $103(2)$ \\
$\mathrm{C}(11)-\mathrm{H}(11) \ldots \mathrm{O}(6)$ & $0.94(2)$ & $2.60(2)$ & $2.758(2)$ & $90(1)$ \\
$\mathrm{C}(18)-\mathrm{H}(18 \mathrm{~A}) \ldots \mathrm{O}(6)$ & $0.99(3)$ & $2.53(2)$ & $2.867(2)$ & $99(2)$ \\
& & & &
\end{tabular}

Symmetry transformations used to generate equivalent atoms: \#1 x,y+1,z 